OPEN ACCESS

Edited by:

Thomas Ruth,

TRIUMF, Canada

Reviewed by:

Alberto Monetti,

Legnaro National Laboratories (INFN),

Cornelia Hoehr

TRIUMF, Canada

*Correspondence:

Reinhard Heinke

reinhard.heinke@cern.ch

Zeynep Talip

zeynep.talip@psi.ch

Specialty section

This article was submitted to

Nuclear Medicine,

a section of the journal

Frontiers in Medicine

Received: 20 May 2021

Accepted: 14 September 2021

Published: 12 October 2021

Citation:

Heinke R, Chevallay E, Chrysalidis $K$

Cocolios TE, Duchemin C

Fedosseev VN, Hurier S, Lambert L,

Leenders B, Marsh BA, van der

Meulen NP, Sprung P, Stora T,

Tosato M, Wilkins SG, Zhang $H$ and Talip Z (2021) Efficient Production of High Specific Activity Thulium-167 at

Paul Scherrer Institute and CERN-MEDICIS

Front. Med. 8:712374

doi: 10.3389/fmed.2021.712374

\title{
Efficient Production of High Specific Activity Thulium-167 at Paul Scherrer Institute and CERN-MEDICIS
}

\begin{abstract}
Reinhard Heinke ${ }^{1,2 *}$, Eric Chevallay ${ }^{2}$, Katerina Chrysalidis ${ }^{2}$, Thomas E. Cocolios ${ }^{1}$, Charlotte Duchemin ${ }^{1,2}$, Valentin N. Fedosseev ${ }^{2}$, Sophie Hurier ${ }^{1,3}$, Laura Lambert ${ }^{2}$, Benji Leenders ${ }^{1,3,4}$, Bruce A. Marsh ${ }^{2}$, Nicholas P. van der Meulen ${ }^{5,6}$, Peter Sprung ${ }^{7}$, Thierry Stora ${ }^{2}$, Marianna Tosato $^{6}$, Shane G. Wilkins ${ }^{2}$, Hui Zhang ${ }^{8}$ and Zeynep Talip ${ }^{6 *}$
\end{abstract}

\footnotetext{
${ }^{1}$ Institute for Nuclear and Radiation Physics, KU Leuven, Leuven, Belgium, ${ }^{2}$ European Organization for Nuclear Research CERN, Geneva, Switzerland, ${ }^{3}$ Belgian Nuclear Research Centre SCK CEN, Mol, Belgium, ${ }^{4}$ Department of

Electromechanical, Systems and Metal Engineering, Ghent University, Ghent, Belgium, ${ }^{5}$ Laboratory of Radiochemistry, Paul Scherrer Institute, Villigen, Switzerland, ${ }^{6}$ Center for Radiopharmaceutical Sciences ETH-PSI-USZ, Paul Scherrer Institute, Villigen, Switzerland, ${ }^{7}$ Analytic Radioactive Materials, Paul Scherrer Institute, Villigen, Switzerland, ${ }^{8}$ Division Large Research Facilities, Paul Scherrer Institute, Villigen, Switzerland
}

Thulium-167 is a promising radionuclide for nuclear medicine applications with potential use for both diagnosis and therapy ("theragnostics") in disseminated tumor cells and small metastases, due to suitable gamma-line as well as conversion/Auger electron energies. However, adequate delivery methods are yet to be developed and accompanying radiobiological effects to be investigated, demanding the availability of ${ }^{167} \mathrm{Tm}$ in appropriate activities and quality. We report herein on the production of radionuclidically pure ${ }^{167} \mathrm{Tm}$ from proton-irradiated natural erbium oxide targets at a cyclotron and subsequent ion beam mass separation at the CERN-MEDICIS facility, with a particular focus on the process efficiency. Development of the mass separation process with studies on stable ${ }^{169} \mathrm{Tm}$ yielded 65 and $60 \%$ for pure and erbium-excess samples. An enhancement factor of thulium ion beam over that of erbium of up to several $10^{4}$ was shown by utilizing laser resonance ionization and exploiting differences in their vapor pressures. Three ${ }^{167} \mathrm{Tm}$ samples produced at the IP2 irradiation station, receiving 22.8 MeV protons from Injector II at Paul Scherrer Institute (PSI), were mass separated with collected radionuclide efficiencies between 11 and 20\%. Ion beam sputtering from the collection foils was identified as a limiting factor. In-situ gamma-measurements showed that up to $45 \%$ separation efficiency could be fully collected if these limits are overcome. Comparative analyses show possible neighboring mass suppression factors of more than 1,000, and overall ${ }^{167} \mathrm{Tm} / \mathrm{Er}$ purity increase in the same range. Both the actual achieved collection and separation efficiencies present the highest values for the mass separation of external radionuclide sources at MEDICIS to date.

Keywords: medical radionuclides, thulium-167, mass separation, laser resonance ionization, MEDICIS, Auger electrons 


\section{INTRODUCTION}

Auger electrons (AEs) can be highly radiotoxic when they decay in the vicinity of DNA in the cell nucleus $(1,2)$, which makes them attractive for radiotherapy. Moreover, recent studies have shown that, even without nuclear localization, AEs can also kill targeted cancer cells by damaging the cell membrane, or non-targeted cells by a local cross-dose effect or a bystander effect (3). Nevertheless, to date, clinical research has been very limited and there is still much to learn about the molecular and cellular radiobiological effects of AEs. Most of the AE-emitting radionuclides also emit conversion electrons with higher energies compared to AEs, resulting in a longer-range effect up to several micrometers $(4,5)$ and less dense ionization.

Many research groups worldwide are focusing on the development of new targeting agents, however, the radionuclides have critical importance for the success of nuclear medicine applications. Previously, it was demonstrated that the decay of ${ }^{125} \mathrm{I}$ in mammalian cell DNA leads to more than one double-strand break per decay (6). Examples of some of the interesting $\mathrm{AE}$ emitting radionuclides include ${ }^{99 \mathrm{~m}} \mathrm{Tc}(7),{ }^{111} \mathrm{In}$ (8), ${ }^{123} \mathrm{I}(9,10),{ }^{125} \mathrm{I}(11),{ }^{201} \mathrm{Tl},{ }^{119} \mathrm{Sb}(12),{ }^{67} \mathrm{Ga}(13),{ }^{191} \mathrm{Pt}$ (14), ${ }^{193 \mathrm{~m}} \mathrm{Pt}$, and ${ }^{195 \mathrm{~m}} \mathrm{Pt}$. Due to the different chemical and pharmacokinetic properties, it is not possible to perform direct preclinical comparison studies with the radionuclides listed above except I and $\mathrm{Pt}$ radionuclides. Up to date, ${ }^{125} \mathrm{I}$ is the most studied AE-emitter (15). Its long half-life (59.4 d) makes it less practical for the clinical applications. Moreover, low radioiodination efficiency $(40-60 \%)$ precludes the kit formulation for the radiopharmaceutical preparation with ${ }^{123} \mathrm{I}$ and ${ }^{125} \mathrm{I}$. Pt isotopes were shown to be highly radiotoxic due to the high emission rates (due to the excess of 30 Auger electrons per decay on average) (5). Nevertheless, low specific activity and no satisfactory radiolabeling strategies obstruct their further investigation.

Thanks to their similar chemical characteristics, such as analogous coordination chemistry, radiolanthanides have great potential to perform basic preclinical comparison studies to improve the understanding of AE therapy. ${ }^{167} \mathrm{Tm}\left(\mathrm{t}_{1 / 2}=9.25 \mathrm{~d}\right)$ is a potential radionuclide both for medical diagnostics and therapy, due to its gamma emission $\left(E_{\gamma}=207.8 \mathrm{keV}\right.$, $I=42 \%)$ for single-photon emission computed tomography (SPECT), and conversion/Auger electron emission $(E=5-$ $100 \mathrm{keV}, I=120 \%$ ) (16). Dosimetry calculations showed a high ratio of absorbed dose rate in the tumor with respect to the normal tissue $(17,18)$. In the past, ${ }^{167} \mathrm{Tm}$-citrate was used for tumor imaging (19) and comparative kinetic studies showed the advantage of ${ }^{167} \mathrm{Tm}$-citrate over simultaneously applied ${ }^{67} \mathrm{Ga}$-citrate in normal and tumor-bearing mice (19, 20). The very fast clearance from blood and good retention in tumor provided justifications for ${ }^{167} \mathrm{Tm}$ to be more appropriate than ${ }^{67} \mathrm{Ga}$. However, suitable targeting methods are still to be developed and accompanying radiobiological effects have to be investigated. This is only possible if this radionuclide becomes available in appropriate activities and quality.
${ }^{167} \mathrm{Tm}$ can be produced via several production routes by charged particle induced reactions (20-24). However, radionuclidically and chemically pure ${ }^{167} \mathrm{Tm}$ batches for extensive studies can only be provided via a combination of mass and chemical separation. Key parameters are the overall efficiency and process duration. Respective operation parameters have to be tailored to the specific peculiarities of the isotope itself, as well as its chemical environment determined by the production path. Systematic preparatory studies are needed for optimization, to avoid losses in each single step, and also due to time constraints imposed by ongoing radioactive decay.

Mass separation at $<100 \mathrm{keV}$ ion beam energy electromagnetic mass separators, including sample preparation and, especially, ion source design and laser ionization system developments, has been proven to work efficiently for lanthanides in recent studies. Efficiency values in the range of a few $10 \%$ and up to more than $50 \%$ were reported for terbium (25), dysprosium (26), holmium (27), ytterbium (28), and lutetium (29) under optimum conditions.

This work focuses on the mass separation process at CERNMEDICIS with ${ }^{167} \mathrm{Tm}$ produced by proton irradiation from natural erbium oxide at PSI. It describes both the preparatory work with stable thulium, as well as the first three collections of ${ }^{167} \mathrm{Tm}$, in detail. As such, it illustrates the first step for the provision of this isotope by the collaboration, but also serves as general description of introducing a new chemical element/target matrix combination in a mass separation facility.

In addition, a full characterization of the samples via gamma-ray spectrometry, ICP-OES and ICP-MS validated their quality grade, toward their use in pre-clinical studies in the near future.

\section{EXPERIMENTAL SETUP AND METHODS}

\subsection{Production of ${ }^{167} \mathrm{Tm}$ at $\mathrm{PSI}$}

${ }^{\text {nat }} \mathrm{Er}_{2} \mathrm{O}_{3}$ target discs, $6 \mathrm{~mm}$ in diameter, were prepared and irradiated at the PSI's IP2 irradiation station (30), using the $72 \mathrm{MeV}$ proton beam from Injector II separated sector cyclotron. Experimental cross-section results of nat $\mathrm{Er}_{2} \mathrm{O}_{3}$ were previously reported by Tarkanyi et al. (31). It was shown that maximum cross-section results were obtained between 18 and $22 \mathrm{MeV}$. In this range, the obtained ${ }^{167} \mathrm{Tm}$ production cross-section results are the sum of the ${ }^{167} \operatorname{Er}(\mathrm{p}, \mathrm{n})$ and ${ }^{168} \operatorname{Er}(\mathrm{p}, 2 \mathrm{n})$ reactions. In the present study, a $2.4 \mathrm{~mm}$ niobium disc was used as a degrader to decrease the proton energy to $22.8 \mathrm{MeV}$. The beam current was set to $50 \mu \mathrm{A}$ and several test irradiations were performed using $30 \mathrm{mg}{ }^{\text {nat }} \mathrm{Er}_{2} \mathrm{O}_{3}$ targets for $8 \mathrm{~h}$. After irradiation, high dose rates were obtained due to the high-energy gamma rays of the coproduct ${ }^{166} \mathrm{Tm}\left(t_{1 / 2}=7.7 \mathrm{~h}\right)$. As a result of dose optimization for transport classification and handling, transports of the samples were performed 7 days after irradiation to await the complete decay of ${ }^{166} \mathrm{Tm}$. Three targets, 106.13 (1,021), 97.81 (929), and $154.37(1,482) \mathrm{MBq}^{167} \mathrm{Tm}$, respectively, at time of shipping, were transported to the CERN-MEDICIS facility for mass separation. Errors are given as 2-sigma interval. 


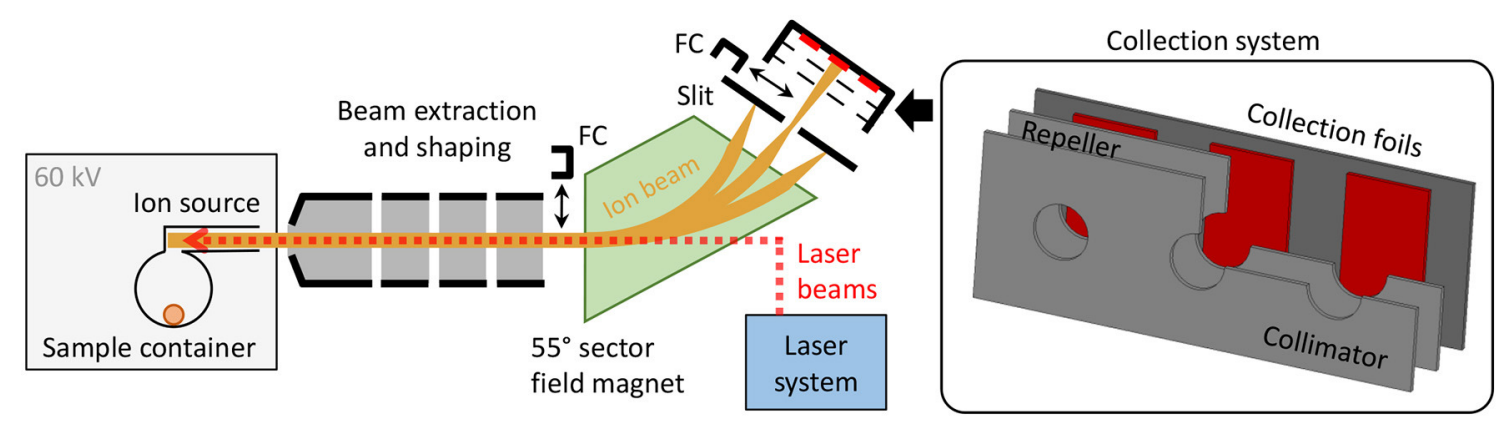

FIGURE 1 | Schematic overview of the MEDICIS mass separator. The inset on the right-hand side schematically depicts a cut view of the actuatable collection system including the sample collection foils.

\subsection{Mass Separation at MEDICIS}

The CERN-MEDICIS (MEDical Isotopes Collected from ISolde) facility is dedicated to the production of non-conventional radionuclides for medical purposes (32-34). As schematically shown in Figure 1, it comprises a $60 \mathrm{kV}$ ion beam dipole sector field magnet mass separator, that uses either targets that are irradiated in a dedicated irradiation station at the adjacent CERN-ISOLDE radioactive ion beam facility (33) by a $1.4 \mathrm{GeV}$ proton beam or, alternatively, radiogenic samples produced and delivered by partner institutes. The radionuclide sample is evaporated and atomized in the ion source, and predominantly ionized by element-selective resonant laser radiation. The ion beam resulting from $60 \mathrm{kV}$ extraction is separated according to the mass-over-charge ratio of its constituents. Faraday cups (FCs) can be inserted into the beam to monitor the beam current before and after separation. Desired ions are implanted into metallic foils situated in a holding system comprising collimator and electron repeller structures to allow for FClike current monitoring. The sample collection system can be actuated during collection to switch between three different implantation foils. The samples are afterwards shipped to the end users for chemical purification and preclinical/clinical tests. A detailed technical overview of the MEDICIS separator, being the principal infrastructure of the presented work, is given in (35). The dedicated MEDICIS resonance ionization laser ion source system MELISSA $(36,37)$, resembling the solid-state laser system of ISOLDE RILIS (38), provides laser beams for element selective ionization by $10 \mathrm{kHz}$ repetition rate wavelength-tunable Ti:sapphire lasers pumped by commercial frequency-doubled diode-pumped Nd:YAG lasers (Innolas Nanio).

For the case of thulium, laser resonance ionization inside a rhenium ion source was realized via a two-step two-resonance scheme developed at the TRILIS group at TRIUMF (39) ${ }^{1}$. A valence electron is excited from the atomic ground state into an intermediate state at $26889.12 \mathrm{~cm}^{-1}$ and, subsequently, into an auto-ionizing state at $51436.78 \mathrm{~cm}^{-1}$. The first step wavelength $\lambda_{1}=371.897 \mathrm{~nm}$ was provided with $500-700 \mathrm{~mW}$, the second

\footnotetext{
${ }^{1}$ Mostamand M, Li R, Romans J, Lassen J. Odd-parity Rydberg and autoionizing states of thulium studies by laser resonance ionization spectroscopy. Spectrochim Acta B. (Submitted).
}

step wavelength $\lambda_{2}=407.371 \mathrm{~nm}$ with around $1.3 \mathrm{~W}$ at the output of the intra-cavity frequency-doubled Ti:sapphire lasers. In the initial setup and optimization phase, it was confirmed that both transitions were well-saturated, as expected from the reported saturation parameters of $\mathrm{P}_{\text {sat }, 1}=12$ (1) $\mathrm{mW}$ and $\mathrm{P}_{\text {sat }, 2}=140$ (21) $\mathrm{mW}$ (39). Additionally, the center wavelength of the resonances was confirmed.

\subsection{Efficiency Assessment With ${ }^{169} \mathrm{Tm}$}

A sample of stable ${ }^{169} \mathrm{Tm}$ was produced by evaporating $10 \mu \mathrm{L}$ of thulium standard solution (Alfa Aesar, 89889 Thulium, AAS standard solution, Specpure ${ }^{\circledR}$, Tm $1,000 \mu \mathrm{g} / \mathrm{mL}$ ) on a rhenium coated tantalum cylinder ("boat"), which was afterwards placed in the center of the MEDICIS target container. This corresponds to an overall amount of $10 \mu \mathrm{g}, 3.56 \times 10^{16}$ atoms or $1,590 \mathrm{nAh}$ integrated charge for singly-charged ions.

The mass separation process efficiency $\epsilon_{\text {sep }}$ of stable isotope separation processes can be determined by comparing the amount of collected atoms of choice $N_{\text {coll }}$ at the end of the process to the amount of these atoms, which were initially present in the target container, $N_{\text {sample }}$. Whereas, the latter is controlled by the amount of calibrated solution that is deposited, the former can be derived from the total collected charge $C_{\text {coll }}$ of the ions at the collection point. This, in turn, can be calculated by timeintegration of the ion current $I_{\text {ion }}$ that is recorded after mass separation in a Faraday cup, where every ion contributes an elementary charge $e$.

$$
\epsilon_{\text {sep }}=\frac{N_{\text {coll }}}{N_{\text {sample }}}=\frac{C_{\text {coll }} / e}{N_{\text {sample }}}=\frac{\int I_{\text {ion }} \mathrm{d} t / e}{N_{\text {sample }}}
$$

For this type of measurement procedure a similar experimental setup on holmium, using neutron activation analysis for sample quantity confirmation (40), quantified the uncertainty in sample preparation to $4 \%$, and the measured efficiencies from ion current were found to be consistent with the actual implanted isotope amount.

This efficiency value contains all single sub-processes that a sample atom has to undergo, i.e., diffusion and effusion out of possible matrix materials, transport into the ion source, transformation and persistence in its atomic form, ionization and 
non-neutralization, extraction, transport through the separator, and detection. A detailed breakdown and disentanglement of the individual contributions requires multiple dedicated experiments, yet, the overall value $\epsilon_{\text {total }}$ also gives a lower limit to each single one, assuming the others close to unity.

\subsection{Efficiency Assessment With ${ }^{169} \mathrm{Tm}$ in Erbium Oxide}

In order to provide a more realistic test environment and to better evaluate the impact of the target material on the aforementioned individual factors involved in the efficiency, a second measurement was performed with the identical target container/ion source assembly. To an identically prepared $1,590 \mathrm{nAh}$ sample of ${ }^{169} \mathrm{Tm}$, a huge excess quantity representative of an irradiated target of nat ${ }^{2} \mathrm{E}_{2} \mathrm{O}_{3}$, used as material for ${ }^{167} \mathrm{Tm}$ production, was added. Therefore, $100 \mathrm{mg}$ of $\mathrm{Er}_{2} \mathrm{O}_{3}$ powder were dissolved in ethanol and the solution deposited and dried on the rhenium boat inside the sample container. This amount of $\mathrm{Er}_{2} \mathrm{O}_{3}$ corresponds to $87 \mathrm{mg}$ of pure erbium, $3.1 \times 10^{20}$ atoms or $14 \mathrm{mAh}$ total charge for singly-charged ions. Thus, the ratio of ${ }^{169} \mathrm{Tm}:{ }^{\text {nat }}$ Er was 1:8,800.

\subsection{Efficiency Assessment With ${ }^{167} \mathrm{Tm}$ From Proton-Irradiated Erbium Oxide}

A series of mass separations on three ${ }^{167} \mathrm{Tm}$ samples, produced as described in section 2.1, was performed at MEDICIS. These samples were weighed as $30-31 \mathrm{mg}$, thus, all containing around $9.4 \times 10^{19}$ erbium atoms, respectively, corresponding to 4.2 $\times 10^{6} \mathrm{nAh}$ for singly-charged ions. The ${ }^{167} \mathrm{Tm}$ activity, after transport of the samples from PSI to MEDICIS and at the start of the actual collections, was 83.04 (799), 76.85 (730), and 122.94 $(1,180) \mathrm{MBq}$, respectively. These activities correspond to contents of around $5 \mathrm{nAh}$, thus, the ${ }^{167} \mathrm{Tm}:{ }^{\text {nat }} \mathrm{Er}$ ratio in all samples was close to $1: 10^{6}$.

Without any further treatment or dissolution, the irradiated erbium pellets were put in the rhenium boat and loaded into the target container. The same container/ion source unit as for the non-radioactive tests was used. The greatly reduced overall amount of thulium compared to the initial tests, in combination with the overwhelming background of surface-ionized erbium on this mass, did not allow for an initial optimization on a Faraday cup. Therefore, a ${ }^{169} \mathrm{Tm}$ tracer amount about 30 -fold more than the radiogenic ${ }^{167} \mathrm{Tm}$ isotope was added in the same way as for the non-radioactive tests. This enabled optimization of the separator and laser operating parameters on mass 169 at still comparatively low container temperatures and thus low evaporation rates, conserving the radioactive sample. For the following actual ${ }^{167} \mathrm{Tm}$ collection phase, the ion current was continuously monitored both on the implantation foil $(0.25 \mathrm{~mm}$ thick gold foil coated with a $500 \mathrm{~nm}$ zinc layer) itself as well as on the collimator cover (diameter $10 \mathrm{~mm}$ ) in front of it. Up to three implantation foils were used per run, while one each was used for internal tests and implanted with low activity.

\subsection{Sample Characterization}

Different techniques were applied to both the initial ${ }^{\text {nat }} \mathrm{Er}_{2} \mathrm{O}_{3}$ targets and the mass-separated samples at MEDICIS and PSI, to characterize and quantify the production and separation process with respect to different quality parameters.

\subsubsection{Activity Measurements}

At PSI, gamma-ray spectrometry measurements were performed using a high-purity germanium (HPGe) detector (Canberra, France). Full energy peak (FEP) efficiency calibration of the spectrometer was performed using a certified ${ }^{152} \mathrm{Eu}$ pointlike source [provided by Physikalisch-Technische Bundesanstalt (PTB), $\left.1.49 \times 10^{5} \mathrm{~Bq}, 01.01 .2015\right]$. The spectra were analyzed with Canberra's Genie 2000 software package. The samples were measured 7 days after irradiation, after mass separation and after dissolving the zinc layer, to determine the mass separation collection efficiencies and the remaining activities on the gold foils, respectively. Half-lives and characteristic gamma-lines of the $\mathrm{Tm}$ radionuclides used for calculations were as follows:

- ${ }^{167} \mathrm{Tm}\left(\mathrm{t}_{1 / 2}: 9.25 \mathrm{~d}, 207.8 \mathrm{keV}(42 \%)(16)\right.$

- ${ }^{168} \mathrm{Tm}\left(\mathrm{t}_{1 / 2}: 93.1 \mathrm{~d}, 815.99 \mathrm{keV}(50.95 \%)(41)\right.$

- ${ }^{165} \mathrm{Tm}\left(\mathrm{t}_{1 / 2}: 30.06 \mathrm{~h}, 242.92 \mathrm{keV}(35.5 \%)(42)\right.$

Deadtime of the measurements was $<6 \%$. The gamma-ray spectrometry measurements were performed with a $300 \mathrm{~cm}$ sample detector distance. Therefore, the geometry of the samples was considered as a point source.

At MEDICIS, the present ${ }^{167} \mathrm{Tm}$ amount in the collection position was determined in-situ during the collection with a compact cadmium zinc telluride (CZT) gamma-ray spectrometry detector GR1 (Kromek, UK) fixed in front of the collection chamber. The device was FEP calibrated with certified sources $\left({ }^{60} \mathrm{Co},{ }^{133} \mathrm{Ba},{ }^{137} \mathrm{Cs},{ }^{152} \mathrm{Eu}\right)$ at a setup resembling the geometry beforehand (43). The count rate on the $207 \mathrm{keV}$ gamma line of ${ }^{167} \mathrm{Tm}$ was utilized. After collection, a dedicated measurement of each foil was performed by the CERN radiation protection service using a HPGe coaxial detector (Mirion Technologies). For absolute efficiency calibration, the ISOCS (In Situ Object Counting System) calibration software (44) was used, which allows to produce an accurate quantitative gamma assay of almost any sample type and size. After the measurements, the Genie 2000 software was used to analyze the acquired spectra and extract the corresponding activities. Corrections regarding counting statistics, detector dead time, systematic uncertainties of the peak area fitting, gamma emission probabilities and sample geometry model uncertainties were considered to determine the errors and limits of detection.

\subsubsection{Isotope Ratio Measurements}

Three ${ }^{167} \mathrm{Tm}$-implanted $\mathrm{Zn}$ coated gold foils were introduced into a reaction vial and the $\mathrm{Zn}$ layer dissolved in $7 \mathrm{~mL} 6 \mathrm{M}$ $\mathrm{HNO}_{3}$. The resultant solution was directly loaded onto a column containing N,N,N/,N/-tetra-n-octyldiglycolamide, nonbranched resin (DGA, particle size 50-100 $\mu \mathrm{m}$, TrisKem International, France; volume $0.08 \mathrm{~mL}$ ), which is based on tetraoctyldiglycolamide as sorbent. The column was rinsed several times with 6.0 $\mathrm{M} \mathrm{HNO}_{3}$ to remove the remaining $\mathrm{Zn}$ from the column. Thulium and erbium were, then, eluted using $5 \mathrm{~mL} 0.05 \mathrm{M} \mathrm{HCl}$ as it was reported in (45). These fractions were analyzed using ICP-MS and ICP-OES techniques. 
A batch of nat ${ }^{2} r_{2} \mathrm{O}_{3}$, the test target and mass-separated samples from three collections were analyzed with the $\mathrm{Nu}$ Instruments Plasma 3 MultiCollector Inductively Coupled Plasma Mass Spectrometer, at PSI's Hot Laboratory, using an Elemental Scientific Apex HF nebulizing system and a self aspiring PFA nebulizer for sample introduction. This Plasma 3 is a sector-field mass spectrometer equipped with 16 Faraday detectors, three Daly detectors, and three secondary electron multipliers, ideal for measuring multiple ion beams simultaneously.

All samples and standards were introduced as acidic solutions in $0.28 \mathrm{M} \mathrm{HNO}_{3}$ and concentration-matched to yield similar ion beam intensities on mass 167 . The online-measured ${ }^{176} \mathrm{Lu} /{ }^{175} \mathrm{Lu}$ ratio of admixed natural $\mathrm{Lu}$ was used to determine the magnitude of instrumental mass discrimination. Post-analyses and analyses of mixed solutions of natural $\mathrm{Er}$ and $\mathrm{Lu}$ were used to characterize the relation between the exponential mass discrimination factors (46) for Er and $\mathrm{Lu}$ [using isotope abundances from (47) and nuclide masses from (48)]. This relation, and the online-obtained exponential mass discrimination factors for $\mathrm{Lu}$, subsequently allowed accurate mass discrimination corrections of Er isotope analyses irrespective of whether $\mathrm{Er}$ in a sample analysis exhibits natural isotope abundances. Ion beam intensities on stable-isotope masses of $\mathrm{Yb}$ and $\mathrm{Dy}$ were monitored for the mathematical correction, assuming natural isotope abundances (47) of isobaric $\mathrm{Yb}$ or Dy contributions to ion beams of $\mathrm{Lu}$ or $\mathrm{Er}$ masses of interest. These corrections were deemed insignificant for all reported results.

For the analyses of mass-separated samples, all ion beams were collected in Faraday detectors connected to amplifier systems having either $10^{12} \Omega$ resistors in the feedback loop of their preamplifier (masses 166 and 168) or $10^{11} \Omega$ resistors (all other masses). The fixed spacing of detectors on the Plasma 3 mass spectrometer did not allow for a simultaneous detection of ${ }^{176} \mathrm{Lu}$ and ${ }^{175} \mathrm{Lu}$ while measuring ion beams at masses 166 and 168 using the more sensitive $10^{12} \Omega$ resistoramplifiers. Therefore, a two-step dynamic analysis routine was chosen, in which the magnetic field was changed 20 times between two $15 \mathrm{~s}$ long measurement steps. Instrumental background signals were corrected using interspersed analyses of sample-and-standard-free $0.28 \mathrm{M} \mathrm{HNO}_{3}$. No isotope ratios involving Er masses 164 or 162 are reported because of Dy contributions to ion beams of these masses that were too high to correct for. Ion beam intensities on mass 167 were ca. $4 \mathrm{pA}$ obtained from $100 \mathrm{ppb}$ (element concentration) solutions of natural Er.

For analyses of the natural $\mathrm{Er}_{2} \mathrm{O}_{3}$ and its irradiated counterpart, all ion beams were detected simultaneously and, with the exception of mass 162, collected in Faraday detectors having either $10^{12} \Omega$ resistors in the feedback loop of their preamplifier ( ${ }^{161} \mathrm{Dy}$ ) or $10^{11} \Omega$ resistors (all other masses). The ion beam at mass 162 was detected using the more sensitive Daly ion counter given the low isotopic abundance of ${ }^{162} \mathrm{Er}$ $[0.139 \%(49)]$. The approximate signal yield of this ion counting detector was determined by adjusting the yield value to obtain the accepted ${ }^{162} \mathrm{Er} /{ }^{167} \mathrm{Er}$ ratio (49), when measuring natural Er. Single measurements consisted of 40 repetitions of $15 \mathrm{~s}$ long signal integrations at ion beam intensities on mass 167 of ca. $2 \mathrm{pA}$ obtained from ca. $50 \mathrm{ppb}$ (element concentration) solutions of natural Er.

The reported Er isotope ratios of the irradiated $\mathrm{Er}_{2} \mathrm{O}_{3}$ were calculated from the \%-deviations of the values obtained on nine bracketing analyses of the natural pre-irradiated $\mathrm{Er}_{2} \mathrm{O}_{3}$ and the accepted isotope composition of natural Er (49) ("bracketing analyses" describe the analyses of natural $\mathrm{Er}_{2} \mathrm{O}_{3}$ that were performed immediately before and after the analyses of the irradiated $\mathrm{Er}_{2} \mathrm{O}_{3}$ ). Final results represent the average of ten individual measurements of the irradiated $\mathrm{Er}_{2} \mathrm{O}_{3}$. The reported uncertainties are the 95\% confidence interval of the reported average values and incorporate the $95 \%$ confidence interval of the corresponding average values of the natural $\mathrm{Er}_{2} \mathrm{O}_{3}$ analyses by quadratic addition. Note that any inaccuracy or drift in the yield value of the Daly ion counting detector canceled out, because the irradiated $\mathrm{Er}_{2} \mathrm{O}_{3}$ analyses were evaluated relative to the composition obtained for the bracketing analyses of the natural pre-irradiated $\mathrm{Er}_{2} \mathrm{O}_{3}$. Instrumental background signals were monitored using interspersed analyses of sample-andstandard-free $0.28 \mathrm{M} \mathrm{HNO}_{3}$, but required no corrections given the relative nature of the data evaluation and the (mostly) orders of magnitude higher ion beam intensities at the most relevant Er masses.

\subsubsection{ICP-OES Measurements}

Standard solutions containing Er, Tm, and Zn (0.1, 0.5, 1, 5 ppm) were prepared in $2 \% \mathrm{HNO}_{3}$ (Merck Suprapur), using SigmaAldrich TraceCERT, 1,000 ppm Er, Tm, and Zn ICP standards. Three mass separated samples were characterized using ICPOES (Agilent ICP-OES 5110) to determine Er, Zn, and Tm concentration of the samples.

\section{RESULTS AND DISCUSSION}

\subsection{Efficiency Assessment With ${ }^{169} \mathrm{Tm}$}

The efficiency determination measurement with the nonradioactive thulium sample, as described in section 2.3, is depicted in Figure 2. Before the start of the measurement, the ion source was set to a nominal operation temperature of around $2,000^{\circ} \mathrm{C}^{2}$. The sample container was gradually heated and the ion current on mass 169 monitored. At a low intensity ion beam of a few $10 \mathrm{pA}$, the operation parameters of the separator and laser system were optimized with direct feedback. Subsequently, the container temperature was increased in steps to investigate the response of the ion signal, which is governed by the supply rate of the atomic fraction into the ion source. Thus, the temperature dependence of the onset of release (around $1,600^{\circ} \mathrm{C}$ for ion currents of $16 \mathrm{nA}$, representing collection of $1 \%$ of the complete sample per hour) and possible interfering chemical reactions or other effects that would decrease the atomic fraction can be probed. Such effects were not observed. After this first phase of heating, the sample gradually evaporated at a fixed temperature

\footnotetext{
${ }^{2}$ Note that this and all further temperature specifications are inferred from optical pyrometer measurements performed after construction of the container/ion source unit and their calibration to the applied current for ohmic heating. For temperatures above $2,000^{\circ} \mathrm{C}$, extrapolation of this calibration is used.
} 


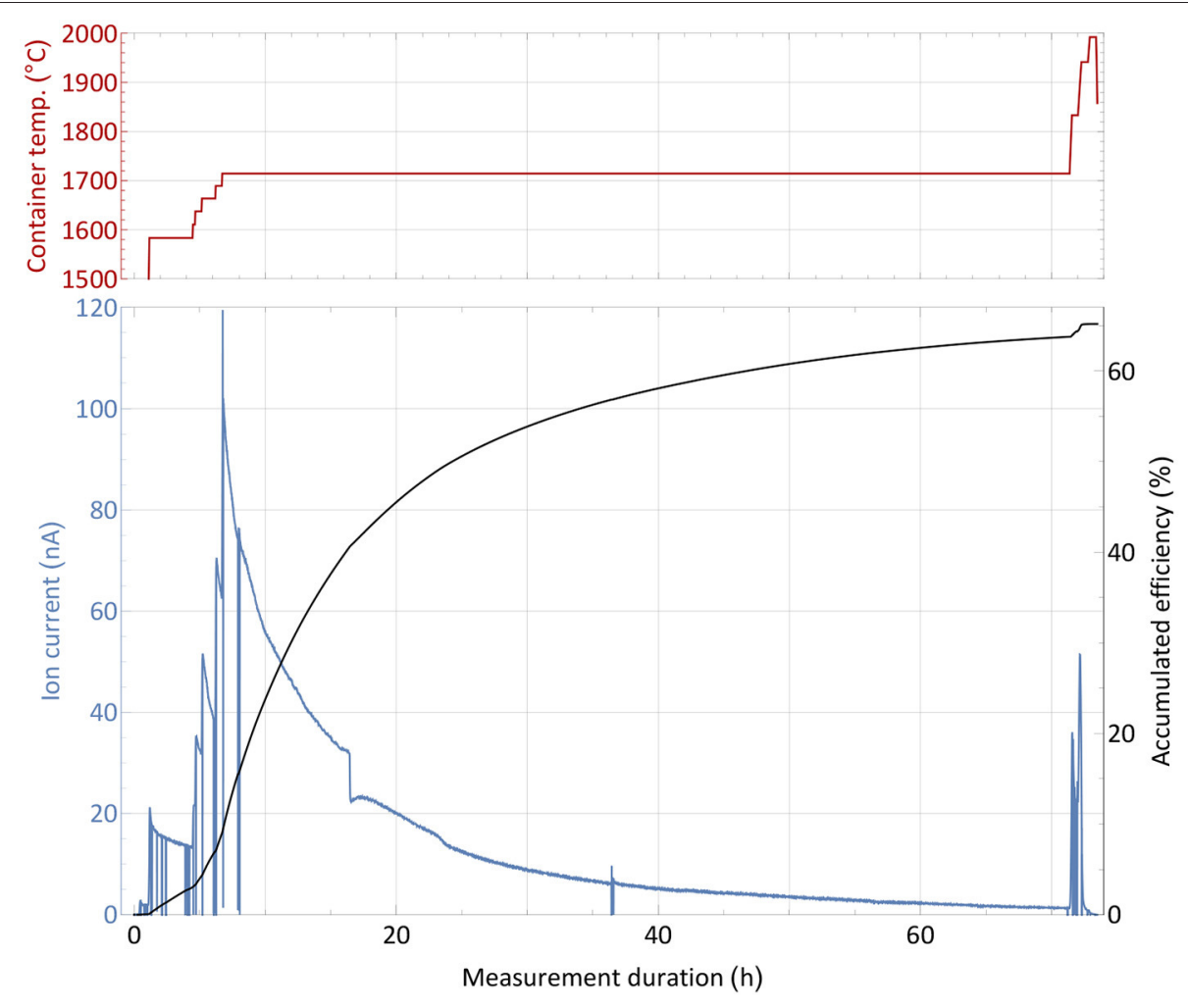

FIGURE 2 | Overall efficiency determination measurement with a 1,590 nAh stable ${ }^{169} \mathrm{Tm}$ sample. The extracted ion current is shown with respect to the sample container temperature (top panel) over time. Additionally, the accumulated efficiency (i.e., the ratio of collected atoms to the initial sample amount) is given for every time point.

of around $1,700^{\circ} \mathrm{C}$, producing a slow exponential decrease in ion current intensity. Through step-wise increase of the temperature, the process of evaporation can be accelerated. Additionally, some parts of the sample which might have condensed at colder spots within the setup were heated out. Once no more significant ion current was detected despite further temperature increase, the sample was treated as depleted and the measurement completed. The chosen overall measurement's duration of 3 days is typical for the collection of radionuclides with a half-life of 5 days or more, such as ${ }^{167} \mathrm{Tm}$. In these collections, the sample heating can be increased gradually over time to allow for less violent outgassing, and decrease total average ion load and stress to the material.

A source of uncertainty in this type of measurement is possible contamination by isobaric ions on the mass of interest, which contribute to the integrated ion current. This would cause an overestimation of the efficiency. Yet, by blocking and unblocking the laser light to the ion source (or changing other laser related parameters as wavelength or pulse timing), sharp, brief drops in ion current can be observed (see Figure 2). The resulting difference in ion current can unambiguously be linked to the element of interest and facilitate the background estimation. During the measurement, this laser enhancement ratio was always $>70$, i.e., the possible contamination portion was below $2 \%$ (and also likely to be surface-ionized thulium itself). Performing a theoretical surface ionization estimation, as described in (50) for a $2,000^{\circ} \mathrm{C}$ hot rhenium source using an estimated survival parameter of $\omega=0.1$ as in (51), the rhenium work function $\Phi=4.72 \mathrm{eV}$ and the thulium ionization potential of $6.1843 \mathrm{eV}$, roughly $0.5 \%$ thulium surface ionization efficiency from the hot cavity itself could be calculated. In combination with recorded laser enhancement factors at certain points of even $>150$, this result is consistent with the $65 \%$ overall efficiency determined by the complete measurement. While this value presents a reliable assessment, it should not be taken as a precise quantification, as a series of measurements would be required to fully determine its uncertainty. Nevertheless, it enqueues into the series of recently achieved efficiencies on lanthanides as described in the introduction and qualified for the subsequent radiogenic Tm separation tests.

\subsection{Efficiency Assessment With ${ }^{169} \mathrm{Tm}$ in Erbium Oxide}

An efficiency and operation characteristics measurement with the large erbium excess sample was performed in analog way as the one described in section 3.1 and is reported in Figure 3. The sharp and brief drops were caused by either probing the laser enhancement factor or inserting a Faraday cup before the separating magnet to probe the total ion beam current emitted from the source. Additionally, records of the mass spectrum 


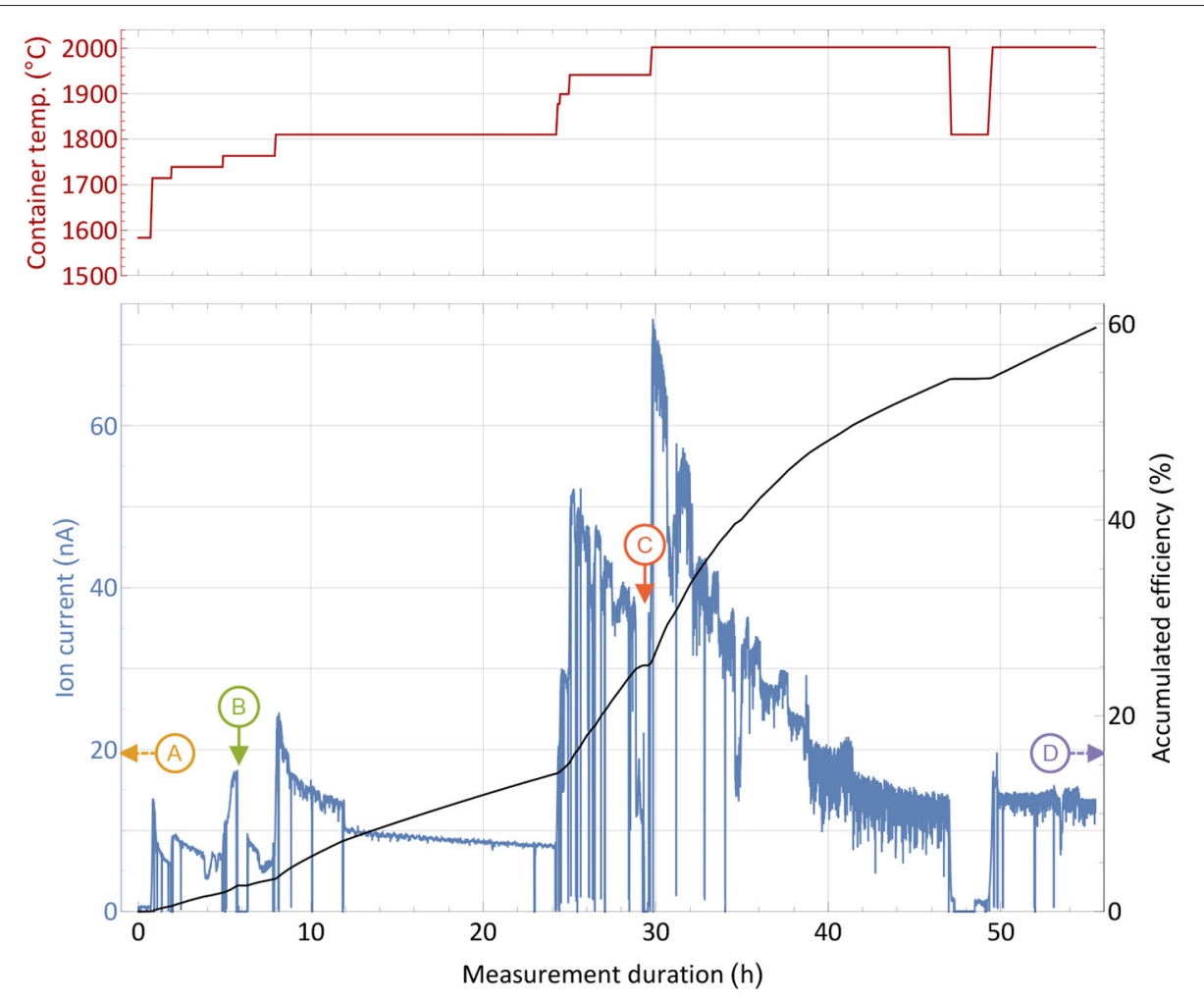

FIGURE 3 | Overall efficiency determination measurement with a 1,590 nAh stable ${ }^{169} \mathrm{Tm}_{\mathrm{m}}$ sample and a 10,000-fold ${ }^{\text {nat }} \mathrm{Er}_{2} \mathrm{O}_{3}$ excess in a presentation analog to Figure 2. Instants where the mass scans presented in Figure $\mathbf{4}$ were taken are marked with capital letters. The extracted ion current is shown with respect to the sample container temperature (top panel) over time.

in the erbium and thulium region were performed at different occasions and certain container temperatures.

From the initial period of the measurement, it was apparent that higher temperatures were required for the same extracted ion current compared to the pure thulium sample. A current of around $16 \mathrm{nA}$, corresponding to $1 \%$ thulium extraction per hour, was reached between 1,700 and $1,800^{\circ} \mathrm{C}$. In order to raise the extracted current to several $10 \mathrm{nA}$ at later stages, $200-300^{\circ} \mathrm{C}$ higher temperature had to be applied. Different reasons may explain this difference: The presence of oxygen and of a more oxidative environment may possibly favor thulium oxide molecule formation, and dissociation of these molecules to required atomic species could occur only at higher temperatures. Indeed, equilibrium chemistry calculations using the HSC Chemistry 10 software confirm higher required temperatures for the same amount of thulium gas, yet also on a lower magnitude of around $50^{\circ} \mathrm{C}$. The latter can only serve as a rough lead though, as equilibrium conditions are not guaranteed, extrapolation of chemical properties for high temperatures are used and the program is not designed to work reliable in low pressure regimes. In addition, a small deviation can be attributed to the exact sample position, but the overall temperature gradient does not exceed $100^{\circ} \mathrm{C}$ over the full container length, and a maximum of $30^{\circ} \mathrm{C}$ for positions near the center, as thermoelectric simulations using ANSYS 2019 R3 show. Finally, the higher ion load due to more surface-ionized erbium in the source can have an influence, leading to a decrease in ionization efficiency of laser-ionized species by providing less confinement in the ion source and thus requiring higher temperature of the setup parts to re-instate the ion survival conditions (50) from the pure thulium sample test. This effect is currently under systematic investigation in view of high throughput laser ion source development.

The most important result is the overall recorded efficiency of $60 \%$, being very close to the value obtained in the pure thulium measurement described in section 2.3. Actually, as seen at the right-hand side of the graph, a considerable thulium ion beam was still present when the machine had to be shut down due to scheduling constraints. An even higher efficiency number for complete depletion of the sample can thus be assumed. This shows that no major interfering effect of the overall evaporation/atomization of thulium by an erbium and oxygen excess exists, besides the shift in temperature which is easy to provide by the setup. Major limitations due to ion load constraints can be neglected as the overall efficiency did not change. The laser enhancement factors varied between 120 in early and 30 in later stages of the measurement.

For the extraction of radioactive ${ }^{167} \mathrm{Tm}$ from erbium oxide, the ion beam on mass 167 is dominated by the stable ${ }^{167} \mathrm{Er}$ isotope, which has a $22.87 \%$ natural abundance. In order to assess 


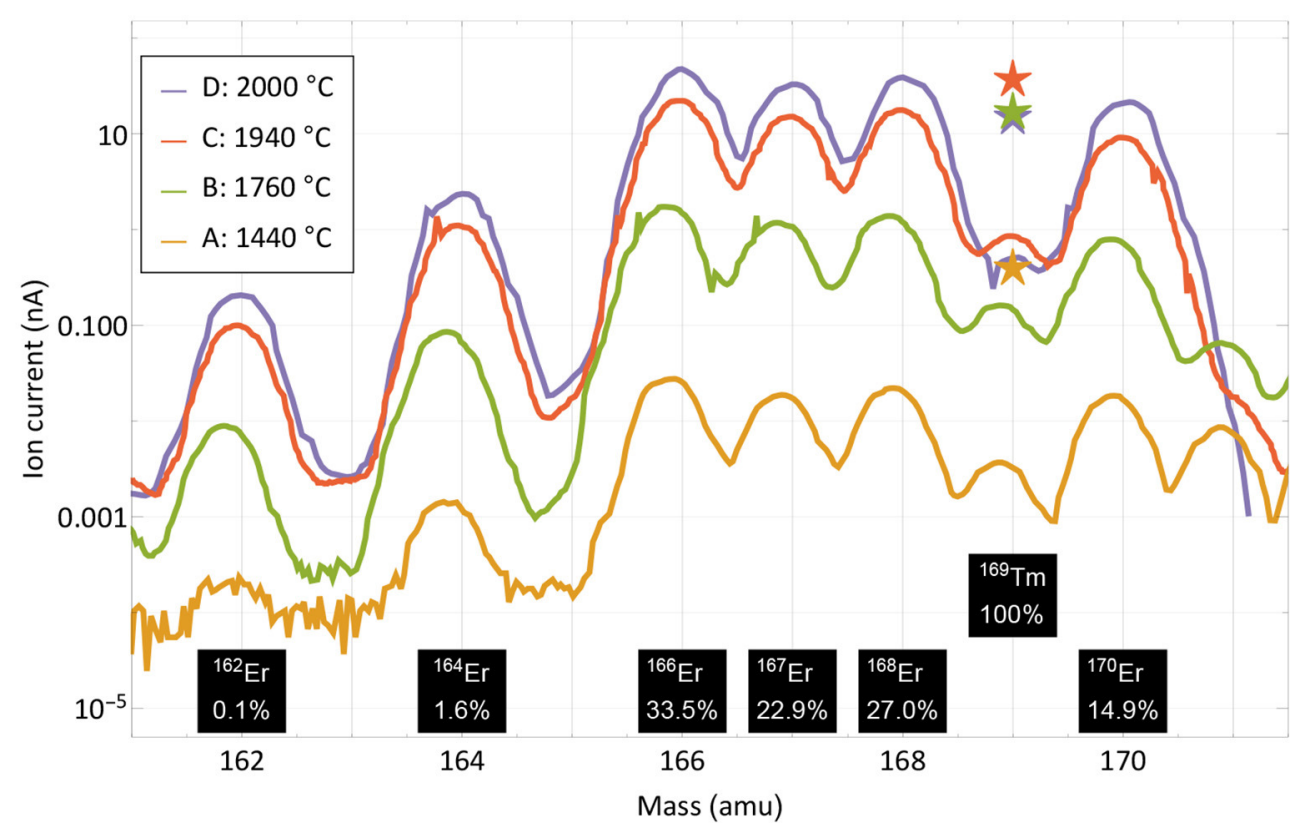

FIGURE 4 | Mass scans of the thulium and erbium region performed at different occasions and temperatures as marked in Figure $\mathbf{3}$. The scans were performed without lasers, the respective measured ion current on mass 169 with lasers is marked with star markers. Natural abundances of erbium and thulium are indicated.

a possible purification on this mass by using the element-selective laser ionization, mass scans of the erbium and thulium region were performed at different occasions during the measurement to determine the current ratio in the extracted ion beam. The measurement points were selected at different temperatures and are marked in Figure 3. Mass scan " $A$ " $\left(1,440^{\circ} \mathrm{C}\right)$ was performed before the efficiency determination measurement at a point with very low thulium evaporation, mass scan " $\mathrm{D}$ " $\left(2,000^{\circ} \mathrm{C}\right)$ directly at the end of the measurement. The mass scan data are shown in Figure 4. These scans were performed without lasers, the star markers on mass 169 indicate the respective measured laser ion current at that point.

The curves show that the separator output on these masses is governed by surface-ionized erbium with its natural abundance pattern of six stable isotopes in between masses 162 and 170. It is also apparent that both the laser enhancement ratio and the ratio of thulium vs. erbium decrease with higher temperatures. Yet, even without laser enhancement, the relative thulium fraction clearly exceeds the sample composition ratio of 1:8,800; thulium extraction is favored over erbium. A breakdown of the involved mechanisms that lead to respective ion beam extraction of erbium and thulium are presented in Figure 5.

Neglecting deviations from the Knudsen law, the evaporation rate of a sample in atomic form is governed by its vapor pressure, which in turn depends on its temperature (52). The thulium vapor pressure exceeds that of erbium by a factor 24060 in the investigated temperature range, with a decreasing trend at higher temperatures (53). It is shown as the base of the theoretical elemental purification factor in Figure 5. Furthermore, the element-selective laser resonance ionization only affects thulium. The ratios shown were extracted from the measured ion currents in Figure 4 on mass 169 with and without lasers and reach from 110 for the first two measurements to 40 and 30 for the last two. The decline in enhancement may be attributed to less pronounced laser ion confinement at higher total ion load (50) and enhanced surface ionization of thulium. For the calculation, it is assumed that the mass 169 ion current without lasers is solely surface-ionized thulium. This assumption is justified from the mass peak shapes shown in Figure 4. For example, on the right-hand side of the mass 170 or left-hand side of the mass 166 erbium peaks, it can be seen that, at a neighboring mass, the remaining tailing has a residual intensity reduced by a factor of close to 1,000 compared to the maximum. Transferring this to mass 169 (where erbium has no stable isotope), the admixture of erbium from the two neighboring peak tailings is significantly $<10 \%$ of its height. Also, no stable isotope of a different element of mass 169 exists. To complete the comparison between thulium and erbium, finally the different surface ionization efficiencies have to be taken into account. Using, again, the model from (50), ionization of erbium is favored by between 60 and $35 \%$ for the investigated temperatures. The red top parts in Figure $\mathbf{5}$ show this factor, which has to be deducted from the previous mechanism all favoring thulium. The expected final overall elemental enrichment factor is then depicted by the dashed horizontal line.

The measured elemental enrichment factors in Figure 5 (lefthand columns) were determined directly by the ratio of the mass 169 thulium ion current (with lasers present) vs. the erbium ion current on mass 167, taking the natural erbium abundances and the sample composition ratio of 1:8,800 into account. These measured values follow the trend of decrease with higher temperature, albeit on an increased level of a factor 


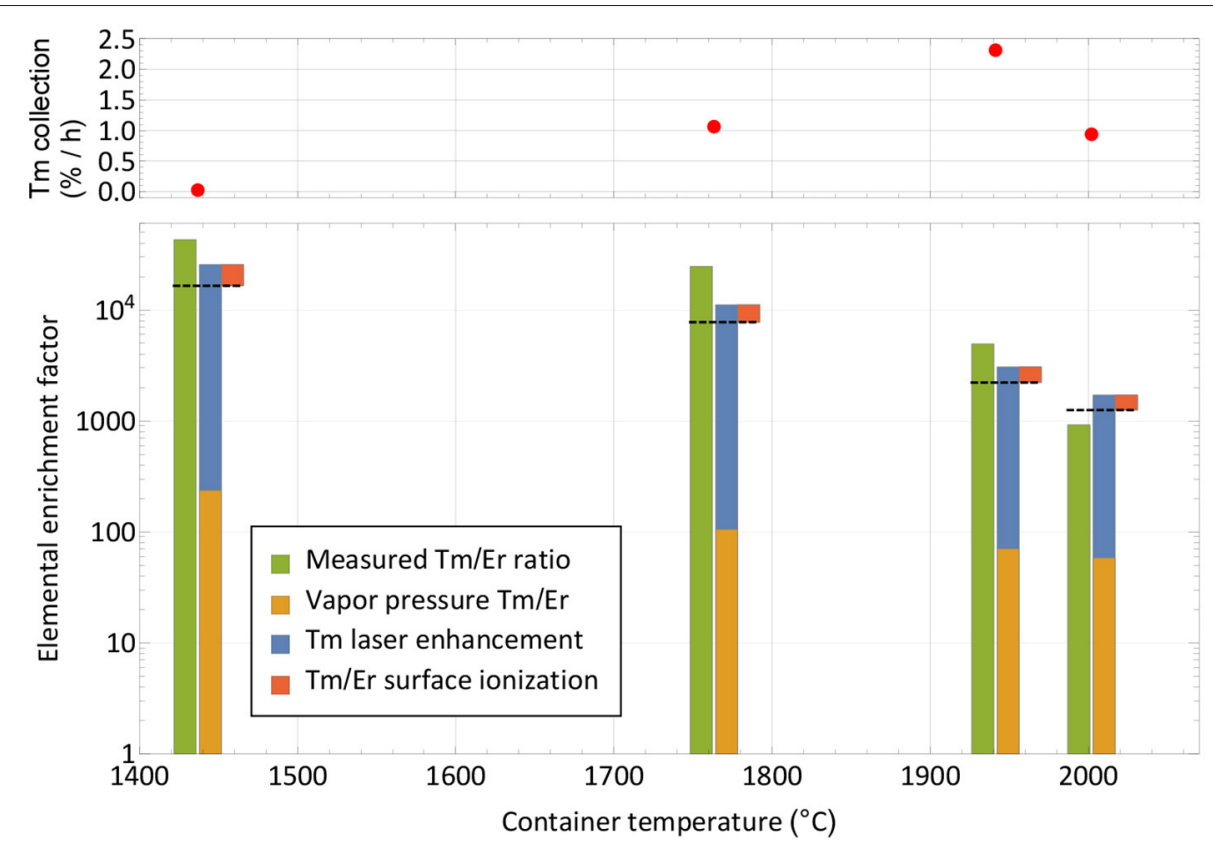

FIGURE 5 | Breakdown of mechanisms involved in the extracted ion beam composition for thulium and erbium. The respective measured elemental enrichment factors (left-hand columns) as extracted from Figure $\mathbf{4}$ are compared with theoretically expected ones (dashed lines) calculated from vapor pressure, laser enhancement (both favoring thulium) and surface ionization efficiency (favoring erbium). The overall trend is reproduced. The top panel depicts the corresponding collection rate at the points of measurement.

2-3 for the first three measurements, and $\approx 0.7$ for the last. For the latter it should be considered that it was performed at the very end of the experiment, where, after already achieving 60\% collection efficiency of the thulium sample, it is close to depletion. In contrast, the erbium should have remained at a higher fraction due to the lower evaporation rate. These investigations, together with the achieved efficiency, hint toward that additional effects not covered in the model that would discriminate thulium in an erbium oxide excess environment are not present. In contrary, molecule formation e.g., on oxide sidebands leading to losses in the atomic form, or permanent adsorption onto the container or ion source material, seem to affect erbium more severely. It should be noted though that on the mono-oxide sideband of erbium the extracted ion current was a factor $>100$ smaller than on the atomic fraction (but the ionization efficiency ratio is unknown), and the calculated surface ionization efficiency for erbium is also consistent with results obtained in (54) for the respective setup. Thus, part of the discrepancy may also be attributed to the rather crude model approach and potential partial inappropriateness in the vapor pressure data for the actual experimental environment.

The resulting measured elemental enrichment factors between 1,000 and a few 10,000 s show the capability of using a mass separator in combination with element-selective (laser resonance) ionization not only for boosting the efficiency and purity of the product itself by mass separation, but also the performance under isobaric contamination. In the presented case, thulium evaporation was favored at lower temperatures, enabling us to perform the collections before the main part of the contaminant is extracted. Should the opposite be the case, a large fraction of the contaminant could be extracted and dumped at low temperatures, while preserving the sample of interest. The limits for both treatments (and especially the latter) are time constraints for the extraction of the sample. This is also depicted in the top panel of Figure 5, where the thulium extraction rate at this point in time is given. Increasing the temperature is mandatory for keeping a reasonable extraction rate over time and achieving full release and depletion of the isotope of interest.

\subsection{Efficiency Assessment With ${ }^{167} \mathrm{Tm}$ From Proton-Irradiated Erbium Oxide}

The collection process of sample number 1 , as described in section 2.5, is shown in Figure 6. The top panels show the stepwise heating of the sample container to successively release the thulium into the ion source. The left-hand bottom graph depicts the corresponding evolution of both the ion current measured on the implantation foil and the rate of ${ }^{167} \mathrm{Tm}$ activity present on the foil. The latter is derived from in-situ gamma-spectroscopic activity determination at certain points in time, which are shown on the right-hand bottom graph. It is complemented by the integrated ion current as measure of overall deposited atoms. Up to three different implantation foils were used per run, one foil each only receiving low activity, which was afterwards used for internal radiochemistry tests and not shipped back to PSI.

Starting from a container temperature of around $1,700^{\circ} \mathrm{C}$ as derived as onset of significant thulium extraction in the separation development (section 3.2), the temperature was 


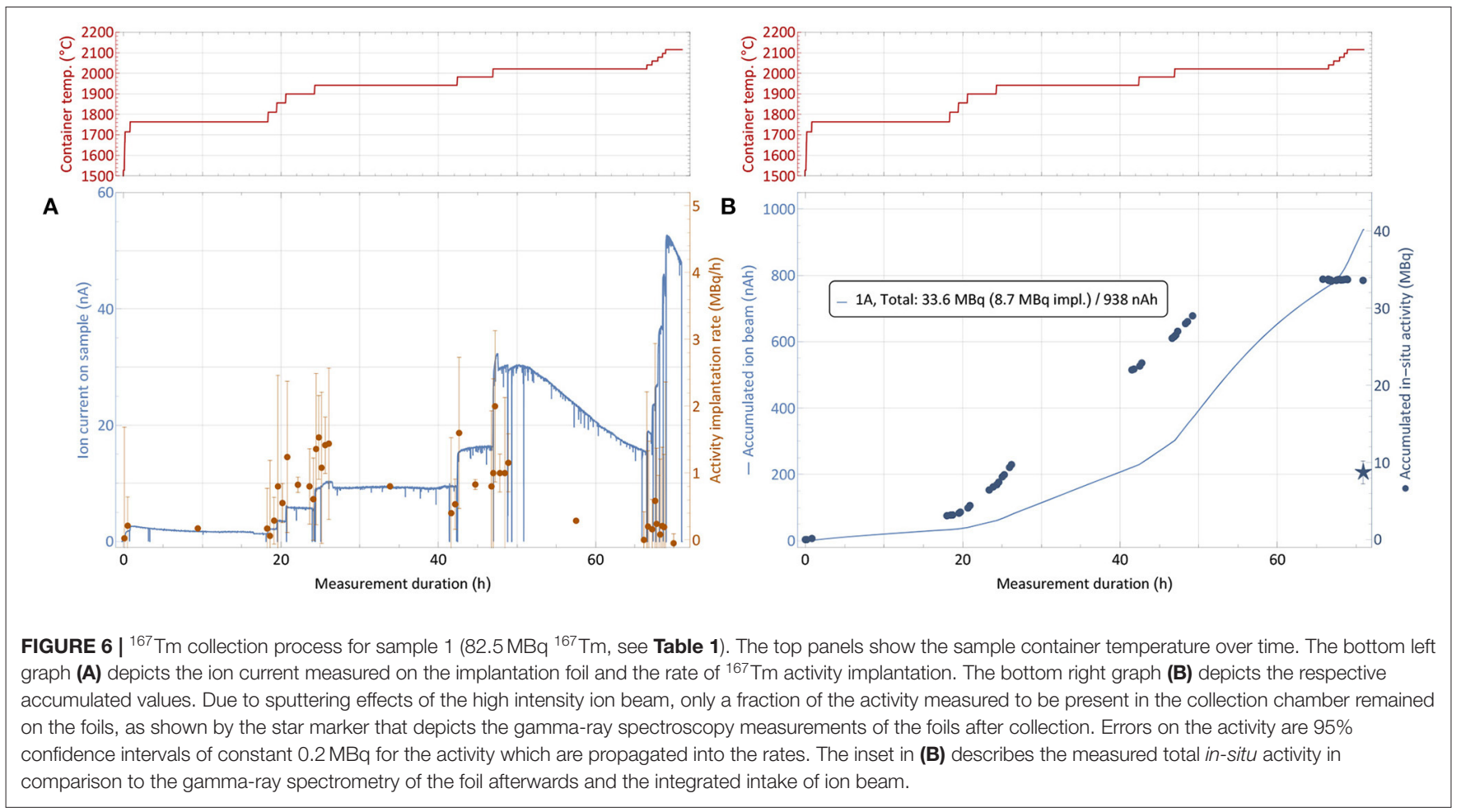

increased further while the amount of activity was measured in regular intervals. Respective activity implantation rates were calculated as shown in Figure 6A, with the errors propagated from the $95 \%$ confidence intervals of $0.2 \mathrm{MBq}$ on the total activity throughout the measurements given by the detector. The point of $1 \%{ }^{167} \mathrm{Tm}$ extraction rate, which was measured at $1,700^{\circ} \mathrm{C}$ with stable ${ }^{167} \mathrm{Tm}_{\mathrm{m}}$ in ${ }^{\text {nat }} \mathrm{Er}_{2} \mathrm{O}_{3}$ environment, was observed to be shifted to roughly $1,800-1,900^{\circ} \mathrm{C}$. These higher required temperature can be caused by the fact that this time the thulium had to diffuse out of the erbium pellet first. Additionally, the even higher excess of erbium and oxygen might shift the effective dissociation point of oxide molecules.

After reaching the onset point of thulium extraction, the container temperature was gradually increased further to evaporate all thulium within the scheduled separation time. As already described in section 3.2 , it can be seen that the ratio of thulium in the extracted mass 167 ion beam (which predominantly consists of surface-ionized stable ${ }^{167} \mathrm{Er}$ ) decreases over time, with sample depletion and with increasing temperature. For ${ }^{167} \mathrm{Tm}$, a collection rate of $1 \mathrm{MBq} / \mathrm{h}$ corresponds to an ion beam current of around $50 \mathrm{pA}$. Thus, in Figure 6A, an identical y-axis height of ion current and activity rate numbers corresponds to $\mathrm{a} \approx 1: 250$ ratio of ${ }^{167} \mathrm{Tm}:{ }^{167} \mathrm{Er}$. Taking the $23 \%$ natural abundance of ${ }^{167} \mathrm{Er}$ into account, this ratio corresponds to an overall ${ }^{167} \mathrm{Tm}:{ }^{\text {nat }} \mathrm{Er}$ purification factor of around 1,000 . Throughout the three collections, actual purification rates between a factor 2 higher (low temperatures) and a factor 10 lower (high temperatures, exhausting thulium sample) than this value were recorded. As expected, these results follow the same trend as in Figure 5, showing no difference in behavior between the test and actual collections. The overall reduction of a factor 10 in comparison with the elemental enrichment rates in the preparatory, non-radioactive test can most probably be attributed to the above mentioned effect of a large fraction of thulium being enclosed inside the erbium pellet, leading to reduced evaporation rates.

The ${ }^{167} \mathrm{Tm}$ separation efficiency as a crucial performance parameter determined by comparing the measured activity on the collection foil to the initial activity in the irradiated sample at the start of the collection. Figure 6B shows the activity at the implantation point for each of the foils from in-situ measurements, determined by the difference between the readout at a given point in time and the background value at the time when this foil was moved into the beam. In previous MEDICIS runs with different elements (from a few up to more than $100 \mathrm{MBq}$ of ${ }^{153} \mathrm{Sm},{ }^{155} \mathrm{~Tb},{ }^{225} \mathrm{Ac}$ ) on identical collection foils (34), this value proved to be a reliable estimate for the gamma-spectrometry measurements that are performed on the foils afterwards by independent measurements in a dedicated, external setup. Yet, for the presented case this value obtained after recuperation of the foil, shown as star marker in Figure 6B with a 95\% confidence interval error band, was significantly lower. Only 8.6 (15) MBq implanted in the main foil were measured, in contrast to $33.6 \mathrm{MBq}$ as estimated from the in-situ determinations. Loss of the main fraction onto the collimator by a fault in the ion optics was excluded by the ratio of respectively measured ion currents, with $<3 \%$ of the overall current recorded on the collimator. An overview of the respective activities and efficiencies for all runs is given in Table 1. 
TABLE 1 | Overview of the three ${ }^{167} \mathrm{Tm}$ collections performed at MEDICIS.

\begin{tabular}{|c|c|c|c|c|c|c|c|c|c|}
\hline \multirow[b]{2}{*}{ No. } & \multirow[b]{2}{*}{$\begin{array}{c}{ }^{167} \text { Tm content }^{a} \\
\text { (MBq) }\end{array}$} & \multirow[b]{2}{*}{ Foil } & \multicolumn{3}{|c|}{ Separated ${ }^{167} \mathrm{Tm}$} & \multicolumn{3}{|c|}{ Collected ${ }^{167} \mathrm{Tm}$} & \multirow[b]{2}{*}{$\begin{array}{c}\text { Ion load } \\
\text { (nAh) }\end{array}$} \\
\hline & & & $\begin{array}{l}\text { Activity per foil } \\
\text { (MBq) }\end{array}$ & $\begin{array}{l}\text { Total run activity } \\
\text { (MBq) }\end{array}$ & Dec.-corr. ${ }^{d}(\%)$ & $\begin{array}{l}\text { Activity per foil } \\
\text { (MBq) }\end{array}$ & $\begin{array}{l}\text { Total run activity } \\
(\mathrm{MBq})\end{array}$ & Dec.-corr. $^{d}(\%)$ & \\
\hline \multirow{2}{*}{1} & \multirow{2}{*}{$83.0(80)$} & $1 \mathrm{~A}$ & 33.6 & \multirow{2}{*}{$33.8 \rightarrow 41 \%$} & \multirow{2}{*}{51} & 8.7 & \multirow{2}{*}{$8.9(15) \rightarrow 11(2) \%$} & \multirow{2}{*}{13} & 938 \\
\hline & & 1B & 0.2 & & & 0.2 & & & 1 \\
\hline \multirow{3}{*}{2} & \multirow{3}{*}{$76.9(73)$} & $2 \mathrm{~A}$ & 14.6 & \multirow{3}{*}{$34.4 \rightarrow 45 \%$} & \multirow{3}{*}{55} & 7.5 & \multirow{3}{*}{$15.4(19) \rightarrow 20(3) \%$} & \multirow{3}{*}{24} & 208 \\
\hline & & $2 \mathrm{~B}$ & 20.2 & & & 7.7 & & & 919 \\
\hline & & $2 \mathrm{C}$ & 0.5 & & & 0.7 & & & 5 \\
\hline \multirow{3}{*}{3} & \multirow{3}{*}{$122.9(118)$} & $3 \mathrm{~A}$ & 28.8 & \multirow{3}{*}{$33.1 \rightarrow 27 \%$} & \multirow{3}{*}{32} & 15.4 & \multirow{3}{*}{$19.2(25) \rightarrow 16(3) \%$} & \multirow{3}{*}{19} & 496 \\
\hline & & 3B & 2.9 & & & 2.9 & & & 45 \\
\hline & & $3 C$ & 4.0 & & & 2.4 & & & 504 \\
\hline
\end{tabular}

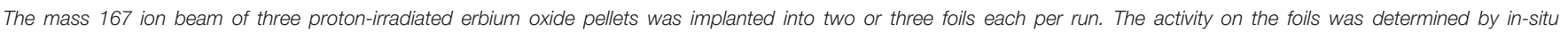

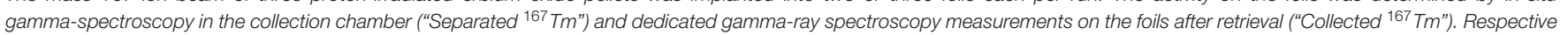
efficiencies and uncertainties where applicable are given and discussed in the text.

${ }^{a}$ At start of collection run.

${ }^{b}$ At end of individual foil implantation.

' Sum of foil activities at end of implantation of the last foil (end of run). Efficiency given as ratio to sample activity at start of run.

a Separation efficiency corrected for radioactive decay.

e Cumulated ion beam intake.

The reason for bold typesetting is to mark these numbers as main result.

The "total run efficiency" describes the ratio of the activity available at the overall end of the run compared to the available activity at its start. If single foils would be extracted directly at the end of their individual implantation (with the "activity per foil" at that time,) instead of all together at the end of the run, a small benefit in avoiding decay losses while implanting the other foils would be gained; thus their sum is higher than the total efficiency. Additionally, the "decay-corrected efficiency" as technical separator performance parameter is given by calculating the theoretical efficiency if no decay was present.

Visual inspection of the foil (Figure 7) and comparison to operation beam parameters (especially ion beam intensity) of previous MEDICIS runs identified sputtering effects on the foil as dominant loss factor. Due to the high ion beam intensity separated on the same mass of collection, some fraction of already implanted ${ }^{167} \mathrm{Tm}$ was released from the foil and condensed onto the structures in the collection chamber. The in-situ gammaactivity measurement was able to detect this fraction (albeit with an unknown, lower efficiency due to a different geometrical distribution further away from the implantation point for which the detector was calibrated-for this reason also error bars on the in-situ activities can not be quantified), which could not be recuperated with the foils any longer: a measurement on the empty foil holder frame after the first implantation yielded 20.8 (36) MBq, and later measurements with the in-situ detector of the empty collection chamber yielded an apparent value between 6 and $7 \mathrm{MBq}$. These results show that the in-situ detector provides a reliable value for the extracted ${ }^{167} \mathrm{Tm}$ activity within the collection chamber and can be used to determine an "extraction and implantation point delivery" efficiency, in contrast to the actual implanted activity remaining on the foils.
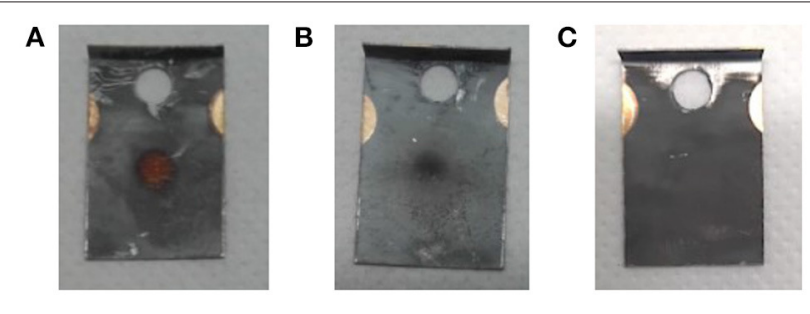

FIGURE 7 | Effect of high ion beam load on the implantation foils. (A) Foil 2B: $919 n A h,(B)$ foil 2A: 208 nAh, (C) foil from different radionuclide implantation with $<30$ nAh ion beam load as comparison.

In order to mitigate this effect with ad-hoc methods in the immediate two subsequent collections, the implantation process was distributed over two foils (in addition to the low-activity one for internal use). The implantation timeline on sample 2 is depicted in Figure 8. While at the end of the first run only around $25 \%$ of the in-situ measured activity was found on the implantation foil, in this configuration fractions of close to $50 \%$ (foil 2A) and 40\% (foil 2B) were achieved. The low-activity implantation foil $2 \mathrm{C}$, which received very limited integrated ion beam exposure, did not show any loss between the in-situ and a dedicated subsequent measurement.

Table 1, where in-situ and actual implanted activities are compared, also gives the overall integrated charge measured on the implantation foils as an easy-to-access metric of ion beam exposure. A complete description would require the time evolution of the ion beam intensity as well. The correlation between higher fractions of activity remaining on the foil and low integrated charge can clearly be established. For run 3 , the ion beam load was distributed as equally as possible on the two 

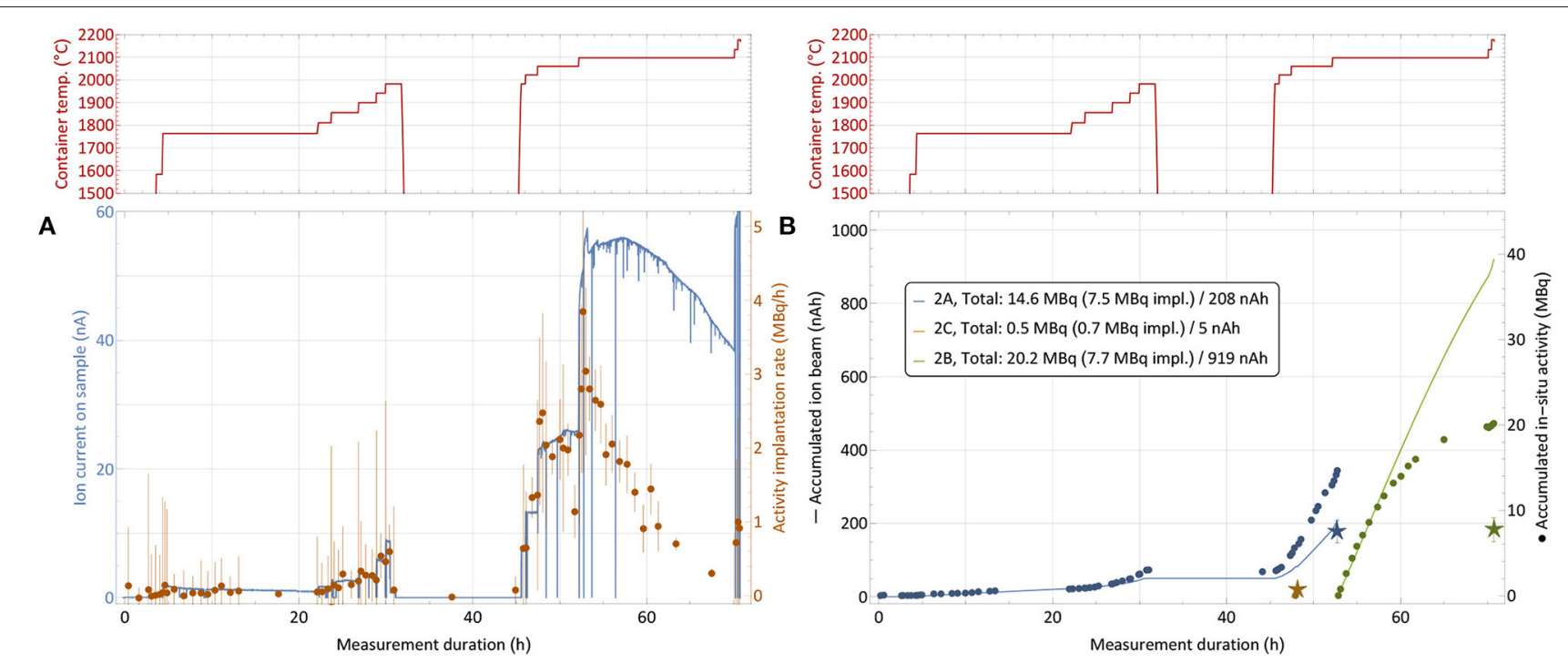

FIGURE 8 $\left.\right|^{167} \mathrm{Tm}$ collection process for sample 2 (76.9 MBq ${ }^{167} \mathrm{Tm}$, see Table 1) with distribution over multiple implantation foils. Depiction analogous to Figure 6. The break between 32 and $45 \mathrm{~h}$ with cooled down container is due to a technical intervention.

available implantation foils (foil 3C was for internal low-activity use), and additionally the beam position was slightly moved at various occasions to avoid exposure of the same spot to the intense beam center for the whole duration. With these measures, in run 2 and 3, 45\% (15.5 out of $34.4 \mathrm{MBq}$ measured in-situ) and $58 \%(19.2 / 33.1)$ of the extracted activity remained on the foil, compared to only $26 \%(8.9 / 33.6)$ for the first run. Followup comparison studies using aluminum and copper layers on the collections foils instead of zinc preliminarily yielded in between 75 and $80 \%$ of in-situ measured activity remaining on the foil after retrieval for aluminum, while in the case of copper only a minor amount of around $10 \%$ was determined for similar ion current exposures.

Taking the extracted ${ }^{167} \mathrm{Tm}$ as performance of the process without implantation into account, these results prove that an extraction and implantation point delivery efficiency of ${ }^{167} \mathrm{Tm}$ from an irradiated ${ }^{\text {nat }} \mathrm{Er}_{2} \mathrm{O}_{3}$ target between 27 and $45 \%$ is possible at MEDICIS if sputtering can be avoided. The decaycorrected efficiency can be compared to the tests with stable ${ }^{169} \mathrm{Tm}$ (60\% efficiency), and achieved values of above $50 \%$ show that no strong additional effects occur in the case of irradiated ${ }^{\text {nat }} \mathrm{Er}_{2} \mathrm{O}_{3}$.

The actual achieved collection efficiency values of $11 \%$, which could be increased to $20 \%$ in subsequent collections by adhoc implementation of sputtering mitigation procedures, already exceed the $5 \%$ goal value on lanthanides for the first stage of MEDICIS and even meet the $20 \%$ in scope of a facility upgrade (32). The influence and mitigation of sputtering effects induced by high intensity beams are currently subject to dedicated developments, including different implantation materials as mentioned above, automated permanent beam movement to avoid a focus on the same spot for the whole duration, and chemical purification of the samples beforehand to remove isobaric contaminants in the matrix.

\subsection{Sample Characterization}

Different techniques were applied on both the initial ${ }^{\text {nat }} \mathrm{Er}_{2} \mathrm{O}_{3}$ targets and the mass-separated samples at MEDICIS and PSI to characterize and quantify the production and separation process with a number of criteria. An overview of the type of measurements performed on specific samples is given in Table 2.

\subsubsection{Activity Measurements}

Representative gamma-ray spectra of the samples before and after mass separation, performed at PSI, are shown in Figure 9. At the time of the measurement, no ${ }^{166} \mathrm{Tm}$ activity was determined due to the 7 days cooling time (Figure 9A).

The activities of the other thulium radionuclides measured for each target were given as follows:

- Target $1\left[{ }^{167} \mathrm{Tm}: 106.13\right.$ (1,021) MBq, ${ }^{168} \mathrm{Tm}: 4.50$ (19) MBq, $\left.{ }^{165} \mathrm{Tm}: 41.04(216) \mathrm{MBq}\right]$

- Target $2\left[{ }^{167} \mathrm{Tm}: 97.81\right.$ (929) MBq, ${ }^{168} \mathrm{Tm}: 8.74$ (29) MBq, ${ }^{165} \mathrm{Tm}: 46.26$ (243) MBq]

- Target $3\left[{ }^{167} \mathrm{Tm}: 154.37(1,482) \mathrm{MBq},{ }^{168} \mathrm{Tm}: 8.67\right.$ (26) MBq, ${ }^{165} \mathrm{Tm}: 61.92$ (325) MBq]

After the mass separation process, the ${ }^{168} \mathrm{Tm}$ activity of the samples could not be determined due to the high ${ }^{167} \mathrm{Tm}$ activity (Figure 9B). Therefore, gamma-ray spectrometry measurements of Sample 2A and 3C were repeated 3 months after the mass separation process (Figure 10). No ${ }^{168} \mathrm{Tm}$ peak was visible for Sample 2A, while $912 \mathrm{~Bq}^{168} \mathrm{Tm}$ was detected in Sample 3C. This activity corresponds to $0.04 \%$ radionuclidic impurity after the mass separation process, and respectively a suppression factor of around 200.

After dissolving the zinc layer of samples $1 \mathrm{~A}, 2 \mathrm{~B}$, and $3 \mathrm{~A}$, the gold foils were re-measured using gamma-ray spectrometry to determine the remaining ${ }^{167} \mathrm{Tm}$ activity on the foils themselves. 
TABLE 2 | Overview of the characterization for different samples.

\begin{tabular}{|c|c|c|c|c|c|}
\hline Samples & Implanted foils & \multicolumn{4}{|c|}{ Characterization of the samples } \\
\hline nat $\mathrm{Er}_{2} \mathrm{O}_{3}$ & & & $x$ & & \\
\hline Test target & & $x$ & $x$ & & \\
\hline Target 1 & $2 \mathrm{~A}$ & $x$ & & & $x$ \\
\hline \multirow[t]{3}{*}{ Target 2} & $2 \mathrm{~B}$ & $x$ & $x$ & $x$ & \\
\hline & $2 \mathrm{C}$ & $x$ & & & \\
\hline & $3 \mathrm{~A}$ & $x$ & $x$ & $x$ & \\
\hline Target 3 & 3B & $x$ & & & \\
\hline
\end{tabular}
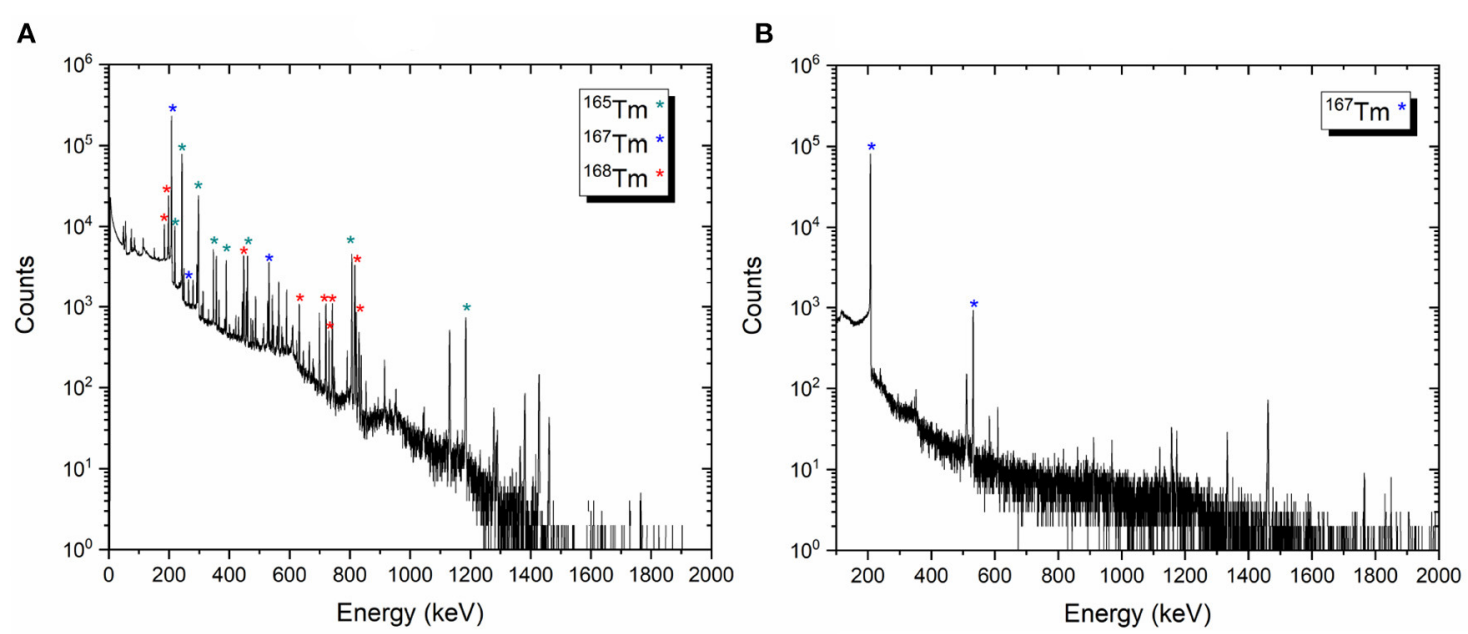

FIGURE 9 | Examples of the gamma-ray spectra of the target material before mass separation process on target 2 (A) and ${ }^{167} \mathrm{Tm}$ implanted foil $2 \mathrm{~A}$ (B).

The results showed $<2 \%$ of the total ${ }^{167} \mathrm{Tm}$ activity at the time of the first gamma-ray spectrometry measurement.

\subsubsection{Isotope Ratio Measurements}

In order to quantify the mass separation power of the process, the relative abundances of erbium isotopes were compared before and after mass separation, i.e., in the proton-irradiated test target and in the foils.

\subsubsection{Natural $\mathrm{Er}_{2} \mathrm{O}_{3}$ and Proton-Irradiated $\mathrm{Er}_{2} \mathrm{O}_{3}$ Samples}

The composition of natural and proton-irradiated $\mathrm{Er}_{2} \mathrm{O}_{3}$ obtained using the mass discrimination correction described in section 2.6.2 are identical within 95\% confidence. Therefore, additional average values using a more precise entirely internal mass discrimination correction based on the measured ${ }^{170} \mathrm{Er} /{ }^{166} \mathrm{Er}$ are also reported for information. An overview is given in Table 3. Note that this correction scheme must fail, introducing artificial deviations from natural isotope ratios, if the ${ }^{170} \mathrm{Er} /{ }^{166} \mathrm{Er}$ of the irradiated $\mathrm{Er}_{2} \mathrm{O}_{3}$ was altered by irradiation.
Using this more precise correction scheme and with the possible exception of ${ }^{164} \mathrm{Er} /{ }^{167} \mathrm{Er}$, all isotope ratios of natural and irradiated $\mathrm{Er}_{2} \mathrm{O}_{3}$ are also identical within the given uncertainties.

\subsubsection{Mass-Separated Samples}

An overview of the isotopic ratios in the implanted foils after mass separation in comparison to the ones in the targets as described above is given in Table 4. For the foils $1 \mathrm{~A}$ and $3 \mathrm{~A}$, the neighboring mass suppression at mass 167 is more than a factor 1,000 , with the exception of ${ }^{168}$ Er suppression being around 600 . These values agree well with the mass peak tailings of the MEDICIS mass separator investigated in section 3.2, Figure 4. For foil $2 \mathrm{~B}$, the respective suppression factors are only between 200 and 300. Post-analyses of the recorded operation parameters of the separator for this run showed no significant change in ion beam shape. A possible explanation is the high ion beam intensity for this implantation at the end of the run whereas the other foils were implanted at the respective beginning, with lower intensity. Sputtering effects of the foil are, thus, more 

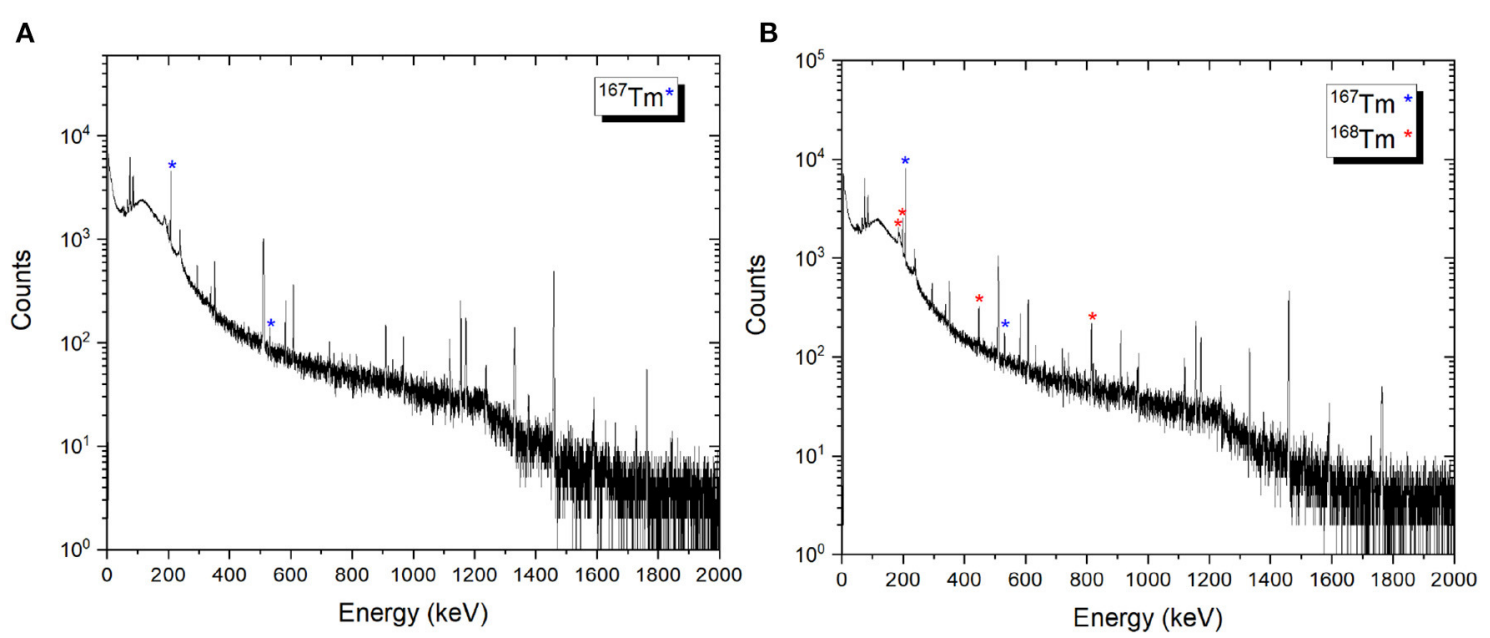

FIGURE 10 | Examples of gamma spectra of the ${ }^{167} \mathrm{Tm}$ implanted foils $2 \mathrm{~A}$ (A) and $3 \mathrm{C}$ (B). The measurements were performed 3 months after mass separation, using the same sample detector distance and counting time.

TABLE 3 | Comparison of the isotope ratios obtained by ICP-MS for natural and proton-irradiated $\mathrm{Er}_{2} \mathrm{O}_{3}$ samples.

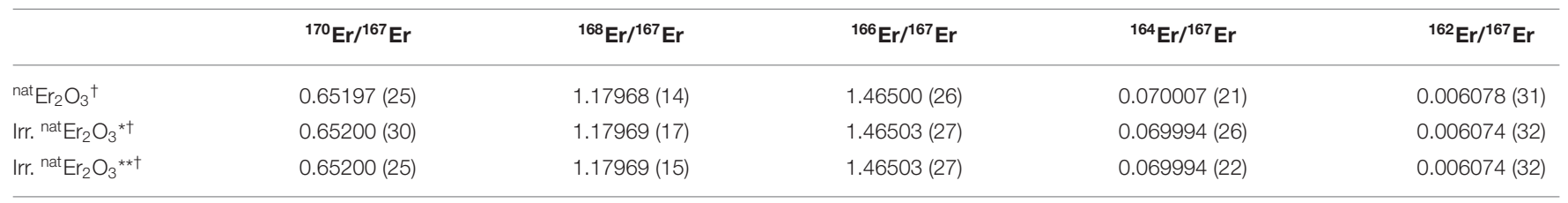

Errors given as $95 \%$ confidence interval (Cl).

${ }^{\dagger}$ From (49), 95\% Cl recalculated using $N=20$.

${ }^{*}$ U Using relation of mass discrimination factors for $\mathrm{Er}$ and admixed Lu from analyses of natural $\mathrm{Er}_{2} \mathrm{O}_{3}$ analyses and including uncertainties from (49).

${ }^{* *}$ Using measured mass discrimination factor from ${ }^{170} \mathrm{Er}{ }^{166} \mathrm{Er}$ and including uncertainties from (49).

TABLE 4 | Comparison of the ICP-MS results of the natural and mass 167 separated samples (1A, 2B, and $3 \mathrm{~A})$.

\begin{tabular}{lccc}
\hline Samples & ${ }^{166} \mathrm{Er} /{ }^{167} \mathrm{Er}$ & ${ }^{168} \mathrm{Er} /{ }^{167} \mathrm{Er}$ & ${ }^{170} \mathrm{Er} /{ }^{167} \mathrm{Er}$ \\
\hline${ }^{n a t} \mathrm{Er}_{2} \mathrm{O}_{3}$ & $1.4650(3)$ & $1.1797(1)$ & $0.6520(2)$ \\
$1 \mathrm{~A}$ & $0.00097(4)$ & $0.00105(3)$ & $0.00034(2)$ \\
$2 \mathrm{~B}$ & $0.0052(2)$ & $0.0060(1)$ & $0.0022(1)$ \\
$3 \mathrm{~A}$ & $0.00141(8)$ & $0.00202(6)$ & $0.00058(3)$ \\
\hline
\end{tabular}

Errors given as 95\% confidence interval.

pronounced, especially at the center of the Gaussian shaped beam. Already implanted ${ }^{167} \mathrm{Er}$ at this spot is removed more strongly than neighboring mass ions tailing in at the sides. As this possible bias induced by position-dependant sputtering in the foil should also be present in the $1 \mathrm{~A}$ and $3 \mathrm{~A}$ foils, albeit less pronounced due to lower ion beam intensity, the extracted neighboring mass suppression factors only present a lower limit to the actual separator performance. The gamma-ray spectrometry results on the ${ }^{168} \mathrm{Tm} /{ }^{167} \mathrm{Tm}$ ratio in foil $3 \mathrm{C}$, also being implanted at a late stage with high ion beam intensity, with a suppression factor of around 200, are in line with these results.
The presence of ${ }^{170} \mathrm{Er}$, with comparable suppression factors as the direct neighboring masses and also a reduced suppression for foil 2B, is unexpected. The mass scans in Figure 4 show no hints of such a tailing over three mass units. Contamination of the foil by other means before the measurements or presence of an unidentified species at mass 170 have to be considered.

\subsubsection{Erbium Content of the Mass-Separated Samples}

After complete decay of ${ }^{167} \mathrm{Tm}$, the $\mathrm{Er}$ and $\mathrm{Zn}$ concentration of the samples $1 \mathrm{~A}, 2 \mathrm{~B}$, and $3 \mathrm{~A}$ were determined using ICP-OES. The results are shown in Table 5 together with the calculated ${ }^{167} \mathrm{Tm}$ amount at time after mass separation.

Isobaric ${ }^{167} \mathrm{Er}$ cannot be mass-separated and is present with a few 100-fold higher quantity. The results are compared with the expected values based on the extracted ${ }^{167} \mathrm{Tm}$ and cumulative ion load $\left({ }^{167} \mathrm{Er}\right)$ as described in section 3.3, yielding similar values for foils $1 \mathrm{~A}$ and $3 \mathrm{~A}$. Foil $2 \mathrm{~B}$ shows some differences between measured and calculated values, although being implanted with the same amount of ions as foil 1A. As discussed above, it was implanted at the end of the run with higher ion beam intensity, apparently causing a stronger loss of Er than Tm under these conditions. Yet, for all foils, the separation increases the 
TABLE 5 | Erbium and zinc remaining traces in the mass separated samples.

\begin{tabular}{|c|c|c|c|c|c|c|c|c|c|c|c|}
\hline \multirow[b]{2}{*}{ Foil } & \multicolumn{5}{|c|}{ Implanted Tm (measured) } & \multicolumn{5}{|c|}{ Extracted Tm (calculated) } & \multirow{2}{*}{$\begin{array}{c}\text { Target } \\
{ }^{167} \mathrm{Tm} / \mathrm{Er} \\
(\mathrm{MBq} / \mu \mathrm{g})\end{array}$} \\
\hline & $\begin{array}{l}\mathrm{Zn} \\
(\mu \mathrm{g})\end{array}$ & $\begin{array}{c}\text { Er } \\
(\mu \mathrm{g})\end{array}$ & $\begin{array}{l}{ }^{167} \mathrm{Tm} \\
(\mathrm{MBq})\end{array}$ & $\begin{array}{c}{ }^{167} \mathrm{Tm} \\
\text { (ng) }\end{array}$ & $\begin{array}{l}{ }^{167} \mathrm{Tm} / \mathrm{Er} \\
(\mathrm{MBq} / \mu \mathrm{g})\end{array}$ & $\begin{array}{c}\text { Er } \\
\text { (nAh) }\end{array}$ & $\begin{array}{c}\mathrm{Er} \\
(\mu \mathrm{g})\end{array}$ & $\begin{array}{l}{ }^{167} \mathrm{Tm} \\
(\mathrm{MBq})\end{array}$ & $\begin{array}{c}{ }^{167} \mathrm{Tm} \\
\text { (ng) }\end{array}$ & $\begin{array}{l}{ }^{167} \mathrm{Tm} / \mathrm{Er} \\
(\mathrm{MBq} / \mu \mathrm{g})\end{array}$ & \\
\hline $1 \mathrm{~A}$ & 0.4 & 1.9 & 8.7 & 2.8 & 4.6 & 938 & 5.8 & 33.6 & 10.7 & 4.6 & $3.1 \times 10^{-3}$ \\
\hline $2 \mathrm{~B}$ & 0.2 & 0.8 & 7.7 & 2.5 & 9.6 & 919 & 5.7 & 20.2 & 6.5 & 3.4 & $2.8 \times 10^{-3}$ \\
\hline $3 A$ & 0.5 & 1.4 & 15.4 & 4.9 & 11.0 & 496 & 3.1 & 28.8 & 9.2 & 9.4 & $4.7 \times 10^{-3}$ \\
\hline
\end{tabular}

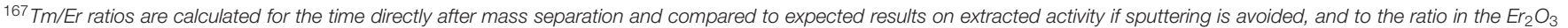
targets before mass separation.

${ }^{167} \mathrm{Tm} / \mathrm{Er}$ ratio compared to the value in the irradiated $\mathrm{Er}_{2} \mathrm{O}_{3}$ targets by a factor of at least 1,500 , being consistent with the enrichment factors obtained as described in sections 3.2 and 3.3. Taking only mass 167 into account (i.e., no mass separation but purification due to element-selective laser ionization and vapor pressure differences), the factor is between 300 and 800 for the investigated foils.

The results obtained from the implanted foils show that additional Er removal after the mass separation process is required to use this solution for radiolabeling of different compounds (in $\mathrm{MBq} / \mathrm{nmol}$ range) to investigate the ${ }^{167} \mathrm{Tm}$ potential with preclinical studies. In addition, ICP-OES results showed that the DGA resin was not sufficient for complete removal of $\mathrm{Zn}$.

\section{SUMMARY AND OUTLOOK}

This work provides a detailed description on the steps followed to establish the mass separation process of external ${ }^{167} \mathrm{Tm}$ samples, produced by proton irradiation of erbium oxide at PSI, at the CERN-MEDICIS facility. Initial tests with non-radioactive thulium samples allowed for optimization of operation parameters and procedures. Separation efficiencies of $65 \%$ for a pure thulium sample and $60 \%$ for a sample with 10,000 -fold erbium excess in these tests proved the adequacy of the setup. The favorable release and ionization of thulium over erbium by utilizing laser resonance ionization and differences in vapor pressures was quantified to reach the regime of $10^{3}-$ $10^{4}$ enrichment factors, and major additional limitations such as undesired molecule formation could be excluded.

Three mass separations to extract ${ }^{167} \mathrm{Tm}$ from protonirradiated erbium oxide pellets in the order of $100 \mathrm{MBq}$ each yielded collection efficiencies between 11 and 20\%. The limiting factor proved to be sputtering of the collected fraction induced by the high intensity erbium fraction in the mass separated ion beam, which was for the first time observed in this extent at MEDICIS. Ad-hoc mitigation procedures were implemented already after the first run. In-situ monitoring of the activity in the collection chamber, thus including the majority of the sputtering losses which were deposited in the direct vicinity, showed that collection efficiencies in the order of $45 \%$ are possible if sputtering can be avoided. Mitigation methods as different implantation materials and automated ion beam movement are under investigation and expected to improve MEDICIS' performance in general. An initial test of aluminum instead of zinc yielded a decrease of sputtering loss to around 20\%. Further tests are planned to be performed using graphite as implantation material. The decay-deconvoluted separation efficiencies with radiogenic ${ }^{167} \mathrm{Tm}$ samples of up $55 \%$ show that no strong additional effects as molecule formation or diffusion phenomena in the target matrix occur in comparison with the cold tests, even though the erbium fraction is even higher. Both the actually implanted $11-20 \%$ as well as the projected $45 \%$ extraction efficiency are to date the highest achieved values with external sources at the MEDICIS facility.

Comparative analyses of the targets and samples before and after mass separation show neighboring mass suppression factors of more than 1,000. Lower values also occur, which might be traced back to sputtering effects as well. The ${ }^{167} \mathrm{Tm} / \mathrm{Er}$ ratio of the samples could be improved by more than a factor of 1,000 by combining mass separation, differences in vapor pressure and selective laser ionization. Yet, isobaric ${ }^{167} \mathrm{Er}$ is still present in a few 100-fold excess and needs to be chemically separated afterwards for application in targeted radionuclide therapy.

The project of providing high specific ${ }^{167} \mathrm{Tm}$ activity for medical research will continue by evaluating alternative production paths. Isotopically enriched erbium oxide, the ${ }^{\text {nat }} \mathrm{Yb}(\mathrm{p}, \mathrm{xn}){ }^{167} \mathrm{Lu} \rightarrow{ }^{167} \mathrm{Tm}$ production route, and spallation of a tantalum target, with respective mass separation process efficiency and sample quality, will be investigated at PSI and at MEDICIS in the near future. The development of accessible and efficient ${ }^{167} \mathrm{Tm}$ production routes will allow the use of this radionuclide for preclinical studies in combination with already developed ligands aimed to be used for the targeting of tumors. Preclinical comparison studies of ${ }^{167} \mathrm{Tm}$ with other Auger electron emitting radiolanthanides such as ${ }^{165} \mathrm{Er}$ and ${ }^{155} \mathrm{~Tb}$ will have a high impact on the understanding of the therapeutic effects of Auger and conversion electrons.

\section{DATA AVAILABILITY STATEMENT}

The raw data supporting the conclusions of this article will be made available by the authors, without undue reservation.

\section{AUTHOR CONTRIBUTIONS}

ZT designed the study. ZT, CD, RH, and TS coordinated the study. EC and LL operated the mass separator. RH, KC, and 
SW operated the laser ionization system. PS performed the ICP-MS analyses. MT assisted the radiochemistry experiments. $\mathrm{HZ}$ optimized the proton beam parameter. $\mathrm{SH}$ performed target unit temperature simulations. BL performed ion source chemistry calculations. $\mathrm{RH}$ and $\mathrm{ZT}$ analyzed the data and drafted the manuscript. TC, VF, BM, NM, and TS provided supervision. All authors discussed and reviewed the manuscript.

\section{FUNDING}

This research received funding from the Swiss National Science Foundation (SNF Grant Number: 200021_188495), the Research Foundation Flanders FWO (Belgium) under contracts FWO SBO Tb-IRMA-V No. S005019N and WO IRI ISOLDE No. I002619N, and the European Union's Horizon 2020 research and innovation

\section{REFERENCES}

1. Ertl HH, Feinendegen LE, Heiniger HJ. Iodine-125, a tracer in cell biology: physical properties and biological aspects. Phys Med Biol. (1970) 15:447. doi: 10.1088/0031-9155/15/3/005

2. Feinendegen LE. Biological damage from the Auger effect, possible benefits. Radiat Environ Biophys. (1975) 12:85-99. doi: 10.1007/BF01328970

3. Ku A, Facca VJ, Cai Z, Reilly RM. Auger electrons for cancer therapy - a review. EJNMMI Radiopharm Chem. (2019) 4:27. doi: 10.1186/s41181-019-0075-2

4. Howell RW. Radiation spectra for Auger-electron emitting radionuclides: report No. 2 of AAPM Nuclear Medicine Task Group No. 6. Med Phys. (1992) 19:1371-83. doi: 10.1118/1.596927

5. Cornelissen B, Vallis KA. Targeting the nucleus: an overview of Augerelectron radionuclide therapy. Curr Drug Discov Technol. (2010) 7:263-79. doi: 10.2174/157016310793360657

6. Kassis AI, Walicka MA. Double-strand break yield following ${ }^{125} \mathrm{I}$ decay effects of DNA conformation. Acta Oncol. (2000) 39:721-6. doi: 10.1080/028418600750063785

7. Tavares AAS, Tavares JMRS. ${ }^{99 m} \mathrm{Tc}$ Auger electrons for targeted tumour therapy: a review. Int J Radiat Biol. (2010) 86:261-70. doi: 10.3109/09553000903564083

8. Teunissen JJM, Kwekkeboom DJ, de Jong M, Esser JP, Valkema R, Krenning EP. Endocrine tumours of the gastrointestinal tract. Peptide receptor radionuclide therapy. Best Pract Res Clin Gastroenterol. (2005) 19:595-616. doi: 10.1016/j.bpg.2005.04.001

9. Smit BS, Slabbert JP, Reinecke SA, Böhm L. Comparison of cell inactivation by Auger electrons using the two reagents 4-123Iiodoantipyrine and 123INaI. Radiat Environ Biophys. (2001) 40:47-52. doi: 10.1007/s004110000083

10. Fourie H, Newman RT, Slabbert JP. Microdosimetry of the Auger electron emitting 123I radionuclide using Geant4-DNA simulations. Phys Med Biol. (2015) 60:3333-46. doi: 10.1088/0031-9155/60/8/3333

11. Adelstein SJ, Kassis AI, Bodei L, Mariani G. Radiotoxicity of iodine- 125 and other auger-electron-emitting radionuclides: background to therapy. Cancer Biother Radiopharm. (2003) 18:301-16. doi: 10.1089/1084978033222 85062

12. Thisgaard H, Jensen M. 119Sb -A potent Auger emitter for targeted radionuclide therapy. Med Phys. (2008) 35:3839-46. doi: 10.1118/1.2963993

13. Othman MFB, Mitry NR, Lewington VJ, Blower PJ, Terry SYA. Re-assessing gallium-67 as a therapeutic radionuclide. Nucl Med Biol. (2017) 46:12-8. doi: 10.1016/j.nucmedbio.2016.10.008

14. Areberg J, Johnsson A, Wennerberg J. In vitro toxicity of ${ }^{191} \mathrm{Pt}$-labeled cisplatin to a human cervical carcinoma cell line (ME-180). Int J Radiat Oncol Biol Phys. (2000) 46:1275-80. doi: 10.1016/S0360-3016(99)00543-X programme under grant agreement No. 101008571 (PRISMAP The European medical radionuclides programme).

\section{ACKNOWLEDGMENTS}

The authors thank the radiation protection teams of PSI (Roman Küng, Andreas Rehmann, and Thomas Lenherr) and CERN (Nabil Menaa, Nicolas Riggaz, Philippe Bertreix, Aurore Boscher, Benjamin Juif, Maxime Munos, Jérémie Comte, Giuseppe Prete, Claire Robin, Blanche Audigier, Elodie Aubert, Alexandre Dorsival, Christelle Saury, Matthieu Deschamps, and Safouane El Idrissi). The authors are grateful to Alexander Vögele for logistic and Roger Geissmann, Alexander Sommerhalder, Marc Ilg, Pascal Grundler, Chiara Favaretto, Muhamet Djelili (PSI) and the PSI-GFA operators for the technical support. The authors thank the ISOLDE and MEDICIS workshop team for preparing the target units and technical assistance.

15. Bodei L, Kassis AI, Adelstein SJ, Mariani G. Radionuclide therapy with iodine125 and other auger-electron-emitting radionuclides: experimental models and clinical applications. Cancer Biother Radiopharm. (2003) 18:861-77. doi: $10.1089 / 108497803322702833$

16. Baglin CM. Nuclear data sheets for $\mathrm{A}=167$. Nucl Data Sheets. (2000) 90:431-644. doi: 10.1006/ndsh.2000.0012

17. Uusijärvi H, Bernhardt P, Ericsson T, Forssell-Aronsson E. Dosimetric characterization of radionuclides for systemic tumor therapy: influence of particle range, photon emission, and subcellular distribution. Med Phys. (2006) 33:3260-9. doi: 10.1118/1.2229428

18. Uusijärvi H, Bernhardt P, Rösch F, Maecke HR, Forssell-Aronsson E. Electronand positron-emitting radiolanthanides for therapy: aspects of dosimetry and production. J Nucl Med. (2006) 47:807-14.

19. Ando A, Ando I, Sakamoto K, Hiraki T, Hisada K, Takeshita M. Affinity of ${ }^{167} \mathrm{Tm}$-citrate for tumor and liver tissue. Eur J Nucl Med. (1983) 8:440-6. doi: 10.1007/BF00252943

20. Beyer GJ, Franke WG, Hennig K, Johannsen BA, Khalkin VA, Kretzschmar $\mathrm{M}$, et al. Comparative kinetic studies of simultaneously injected ${ }^{167} \mathrm{Tm}$ - and ${ }^{67} \mathrm{Ga}-\mathrm{Citrate}$ in Normal and Tumour bearing mice. Int J Appl Radiat Isotopes. (1978) 29:673-81. doi: 10.1016/0020-708X(78)90105-9

21. Tárkányi F, Hermanne A, Király B, Takács S, Ignatyuk AV. Study of excitation functions of alpha-particle induced nuclear reactions on holmium for ${ }^{167} \mathrm{Tm}$ production. Appl Radiat Isotopes. (2010) 68:404-11. doi: 10.1016/j.apradiso.2009.11.043

22. Homma Y, Sugitani Y, Matsui Y, Matsuura K, Kurata K. Cyclotron production of ${ }^{167} \mathrm{Tm}$ from natural erbium and natural holmium. Int J Appl Radiat Isotopes. (1980) 31:505-8. doi: 10.1016/0020-708X(80)90314-2

23. Tárkányi F, Hermanne A, Takács S, Király B, Spahn I, Ignatyuk AV. Experimental study of the excitation functions of proton induced nuclear reactions on ${ }^{167} \mathrm{Er}$ for production of medically relevant ${ }^{167} \mathrm{Tm}$. Appl Radiat Isotopes. (2010) 68:250-5. doi: 10.1016/j.apradiso.2009.10.043

24. Hermanne A, Rebeles RA, Tárkányi F, Takács S, Spahn I, Ignatyuk AV. High yield production of the medical radioisotope ${ }^{167} \mathrm{Tm}$ by the ${ }^{167} \operatorname{Er}(\mathrm{d}, 2 \mathrm{n})$ reaction. Appl Radiat Isotopes. (2011) 69:475-81. doi: $10.1016 /$ j.apradiso.2010.10.003

25. Gadelshin VM, Formento-Cavaier R, Haddad F, Heinke R, Stora T, Studer $\mathrm{D}$, et al. Terbium medical isotope production: laser resonance ionization scheme development. Front Med. (2021) 8:727557. doi: 10.3389/fmed.2021. 727557

26. Studer D, Dyrauf P, Naubereit P, Heinke R, Wendt K. Resonance ionization spectroscopy in dysprosium. Hyperfine Interact. (2017) 238:1-11. doi: 10.1007/s10751-016-1384-4

27. Kieck T, Dorrer H, Düllmann CE, Gadelshin V, Schneider F, Wendt K. Highly efficient isotope separation and ion implantation of ${ }^{163} \mathrm{Ho}$ for the 
ECHo project. Nucl Instrum Methods Phys Res Sect A. (2019) 945:162602. doi: 10.1016/j.nima.2019.162602

28. Alkhazov GD, Batist LK, Bykov AA, v d Vitman, Letokhov VS, Mishin VI, et al. Application of a high efficiency selective laser ion source at the IRIS facility. Nucl Instrum Methods Phys Res Sect A. (1991) 306:400-2. doi: 10.1016/0168-9002(91)90348-T

29. Gadelshin VM, Heinke R, Kieck T, Kron T, Naubereit P, Rösch F, et al. Measurement of the laser resonance ionization efficiency for lutetium. Radiochim Acta. (2019) 107:653-61. doi: 10.1515/ract-2019-3118

30. Grundler PV, Eichler R, Talip Z, Schubiger PA, Schibli R, van der Meulen NP. The metamorphosis of radionuclide production and development at Paul Scherrer Institute. Chimia. (2020) 74:968-75. doi: 10.2533/chimia.2020.968

31. Tárkányi F, Takács S, Hermanne A, Ditrói F, Király B, Baba M, et al. Study of activation cross sections of proton induced reactions on erbium for practical applications. Nucl Instrum Methods Phys Res Sect B. (2008) 266:4872-6. doi: 10.1016/j.nimb.2008.08.005

32. dos Santos Augusto R, Buehler L, Lawson Z, Marzari S, Stachura M, Stora $\mathrm{T}$, et al. CERN-MEDICIS (medical isotopes collected from ISOLDE): a new facility. Appl Sci. (2014) 4:265-81. doi: 10.3390/app4020265

33. Duchemin C, Ramos JP, Stora T, editors. CERN-MEDICIS: A Unique Facility for the Production of Non-Conventional Radionuclides for the Medical Research. Geneva: JACoW Publishing (2020).

34. Duchemin C, Ramos JP, Stora T, Ahmed E, Aubert E, Audouin N, et al. CERNMEDICIS: a review since commissioning in 2017. Front Med. (2021) 8:693682. doi: 10.3389/fmed.2021.693682

35. Martinez Palenzuela Y, et al. The CERN-MEDICIS isotope separator beamline. Front Med. (2021) 8:689281. doi: 10.3389/fmed.2021.689281

36. Gadelshin VM, Wilkins S, Fedosseev VN, Barbero E, Barozier V, Bernardes AP, et al. First laser ions at the CERN-MEDICIS facility. Hyperf Interact. (2020) 241:1-9. doi: 10.1007/s10751-020-01718-y

37. Gadelshin VM, Barozier V, Cocolios TE, Fedosseev VN, Formento-Cavaier $\mathrm{R}$, Haddad F, et al. MELISSA: laser ion source setup at CERN-MEDICIS facility. Blueprint. Nucl Instrum Methods Phys Res Sect B. (2020) 463:460-3. doi: 10.1016/j.nimb.2019.04.024

38. Fedosseev V, Chrysalidis K, Goodacre TD, Marsh B, Rothe S, Seiffert C, et al. Ion beam production and study of radioactive isotopes with the laser ion source at ISOLDE. J Phys G. (2017) 44:084006. doi: 10.1088/1361-6471/aa78e0

39. Mostamand M. Laser developments and study of Rydberg and autoionizing Rydberg states in Tm, La and At using resonant ionization laser spectroscopy [Ph.D. thesis]. University of Manitoba, Vancouver, BC, Canada (2020). Available online at: http://hdl.handle.net/1993/34548

40. Schneider F, Chrysalidis K, Dorrer H, Düllmann CE, Eberhardt K, Haas $\mathrm{R}$, et al. Resonance ionization of holmium for ion implantation in microcalorimeters. Nucl Instrum Methods Phys Res Sect B. (2016) 376:388-92. doi: 10.1016/j.nimb.2015.12.012

41. Baglin CM. Nuclear data sheets for $\mathrm{A}=168$. Nucl Data Sheets. (2010) 111:1807-2080. doi: 10.1016/j.nds.2010.07.001

42. Jain AK, Ghosh A, Singh B. Nuclear data sheets for A $=165$. Nucl Data Sheets. (2006) 107:1075-346. doi: 10.1016/j.nds.2006.05.002

43. Christodoulou P. An in-situ gamma-spectrometry system for the characterization of non-conventional radionuclides for medical research [M. Sc. thesis]. Complutense University of Madrid, Madrid, Spain (2020). Available online at: https://cds.cern.ch/record/2732064

44. Bronson FL, Young B. Mathematical calibration of Ge detectors, and the instruments that use them. In: Proceedings of 5th Annual NDA/NDE Waste
Characterization Conference. (1997). Available online at: https://www.osti. gov/biblio/539248

45. Talip Z, Borgna F, Müller C, Ulrich J, Duchemin C, Stora T, et al. Production of mass separated erbium-169 towards the first preclinical in vitro investigations. Front Med. (2021) 8:479. doi: 10.3389/fmed.2021. 643175

46. Russell WA, Papanastassiou DA, Tombrello TA. Ca isotope fractionation on the Earth and other solar system materials. Geochim Cosmochim Acta. (1978) 42:1075-90. doi: 10.1016/0016-7037(78)90105-9

47. Meija J, Coplen TB, Berglund M, Brand WA, de Biévre P, Gröning $\mathrm{M}$, et al. Isotopic compositions of the elements 2013 (IUPAC Technical Report). Pure Appl Chem. (2016) 88:293-306. doi: 10.1515/pac-20150503

48. Audi G, Wang M, Wapstra AH, Kondev FG, MacCormick M, Xu X, et al. The Ame2012 atomic mass evaluation. Chinese Phys C. (2012) 36:1287-602. doi: $10.1088 / 1674-1137 / 36 / 12 / 002$

49. Chang TL, Zhao MT, Li WJ, Wang J, Qian QY. Absolute isotopic composition and atomic weight of erbium. Int J Mass Spectrom. (1998) 177:131-6. doi: 10.1016/S1387-3806(98)14057-5

50. Kirchner R. On the thermoionization in hot cavities. Nucl Instrum Methods Phys Res Sect A. (1990) 292:203-8. doi: 10.1016/0168-9002(90)90377-I

51. Day Goodacre T, Chrysalidis K, Fedorov DV, Fedosseev VN, Marsh BA, Molkanov PL, et al. The identification of autoionizing states of atomic chromium for the resonance ionization laser ion source of the ISOLDE radioactive ion beam facility. Spectrochim Acta Part B. (2017) 129:58-63. doi: 10.1016/j.sab.2017.01.001

52. Eichler B, Hübener S, Erdmann N, Eberhardt K, Funk H, Herrmann G, et al. An atomic beam source for actinide elements: concept and realization. Radiochim Acta. (1997) 79:221-3. doi: 10.1524/ract.1997.79.4.221

53. Honig RE, Kramer DA. Vapor Pressure Curves of the Elements. Princeton, NJ: RCA Laboratories. (1968).

54. Formento-Cavaier R, Köster U, Crepieux B, Gadelshin VM, Haddad F, Stora T, et al. Very high specific activity erbium ${ }^{169} \mathrm{Er}$ production for potential receptor-targeted radiotherapy. Nucl Instrum Methods Phys Res Sect B. (2020) 463:468-71. doi: 10.1016/j.nimb.2019.04.022

Conflict of Interest: The authors declare that the research was conducted in the absence of any commercial or financial relationships that could be construed as a potential conflict of interest.

Publisher's Note: All claims expressed in this article are solely those of the authors and do not necessarily represent those of their affiliated organizations, or those of the publisher, the editors and the reviewers. Any product that may be evaluated in this article, or claim that may be made by its manufacturer, is not guaranteed or endorsed by the publisher.

Copyright (๑) 2021 Heinke, Chevallay, Chrysalidis, Cocolios, Duchemin, Fedosseev, Hurier, Lambert, Leenders, Marsh, van der Meulen, Sprung, Stora, Tosato, Wilkins, Zhang and Talip. This is an open-access article distributed under the terms of the Creative Commons Attribution License (CC BY). The use, distribution or reproduction in other forums is permitted, provided the original author(s) and the copyright owner(s) are credited and that the original publication in this journal is cited, in accordance with accepted academic practice. No use, distribution or reproduction is permitted which does not comply with these terms. 